\title{
Trace Operator for 2-microlocal Besov Spaces with Variable Exponents
}

\author{
Takahiro NOI \\ Chuo University \\ (Communicated by K. Yoshitomi)
}

\begin{abstract}
Moura, Neves and Schneider proved the trace theorem for 2-microlocal Besov spaces with variable integrability and smoothness, where the summability parameter was constant (Math. Nachr., 286 (2013) 1240-1254). In this paper, we extend the trace theorem for the case that the summability parameter is also a variable exponent.
\end{abstract}

\section{Introduction}

The 2-microlocal Besov spaces and Trieble-Lizorkin spaces with variable exponents were introduced by Kempka [7, 8] who studied the atomic, moleculer and wavelet decompositions for the spaces, but the summability parameter was assumed to be constant on the 2-microlocal Besov space with variable exponents case. Moura, Neves and Schneider [12] studied the trace theorem for 2-microlocal Besov spaces with variable exponents by using atomic decompositions, but the summability parameter is constant. Kempka and Vybíral [10] have introduced 2-microlocal Besov spaces with variable exponents in the case where summability parameter is also a variable exponent, and have studied local mean characterization.

The goal of this paper is to prove the trace theorem for 2-microlocal Besov spaces with variable exponents in the cases where the summability parameter is also a variable exponent. To do this, we consider an atomic decomposition for 2-microlocal Besov spaces with variable exponents.

In this paper, we use the following notations :

- If there exists a positive constant $c$ independent of $A$ and $B$ such that $A \leq c B$ holds, then we write $A \lesssim B$.

- $A \gtrsim B$ means $B \lesssim A$.

- If $A \lesssim B$ and $B \lesssim A$, then we write $A \sim B$.

- For $v \in \mathbb{Z}$ and $m=\left(m_{1}, m_{2}, \ldots, m_{n}\right) \in \mathbb{Z}^{n}$, we define $Q_{v m}:=\prod_{k=1}^{n}\left[\frac{m_{k}}{2^{v}}, \frac{m_{k}+1}{2^{v}}\right)$.

Received March 20, 2015; revised November 4, 2015

Mathematics Subject Classification: 42B35, 42B25, 46E30

Key words and phrases: 2-microlocal Besov spaces, Atomic decomposition, Trace operator, variable exponent 
- Let $\chi_{v m}$ be the characteristic function on $Q_{v m}$.

- Let $\gamma \in \mathbb{R}$ be positive. Then $\gamma Q_{v m}$ is the cube in $\mathbb{R}^{n}$ concentric with $Q_{\nu m}$ and with side length $\gamma$ times the side length of $Q_{v m}$.

- Let $a, b \in \mathbb{Z}$, then $\delta_{a, b}$ is the Kronecker delta:

$$
\delta_{a, b}= \begin{cases}1 & a=b, \\ 0 & a \neq b .\end{cases}
$$

To close this section, we describe how we organize the remaining part of this paper. This paper consists of 8 Sections as follows. In Section 2, we define the 2-microlocal Besov spaces with variable exponents $B_{p(\cdot), q(\cdot)}^{\boldsymbol{w}}\left(\mathbb{R}^{n}\right)$ and state local mean characterization for $B_{p(\cdot), q(\cdot)}^{\boldsymbol{w}}\left(\mathbb{R}^{n}\right)$ (Theorem 1). Next we introduce fundamental results on the variable exponents analysis in Section 3. In Section 4, we prove an atomic decomposition for $B_{p(\cdot), q(\cdot)}^{w}\left(\mathbb{R}^{n}\right)$. To show that $B_{p(\cdot), q(\cdot)}^{\boldsymbol{w}}\left(\mathbb{R}^{n}\right)$ is a quasi-Banach space, we prove a version of the Fatou lemma for $B_{p(\cdot), q(\cdot)}^{w}\left(\mathbb{R}^{n}\right)$. In order to prove that $\mathcal{S}\left(\mathbb{R}^{n}\right)$ is dense in $B_{p(\cdot), q(\cdot)}^{\boldsymbol{w}}\left(\mathbb{R}^{n}\right)$ (Theorem 9), we apply a multiplier theorem (Theorem 6). In Section 5, we prove the multiplier theorem (Theorem 6). We apply the atomic decomposition theorem and the fact that $\mathcal{S}\left(\mathbb{R}^{n}\right)$ is dense in $B_{p(\cdot), q(\cdot)}^{\boldsymbol{w}}\left(\mathbb{R}^{n}\right)$ to prove a trace theorem (Theorem 10) . To do this we show that $\mathcal{S}\left(\mathbb{R}^{n}\right)$ is dense in $B_{p(\cdot), q(\cdot)}^{w}\left(\mathbb{R}^{n}\right)$ (Theorem 9) in Section 7. Finally, we state the trace theorem (Theorem 10) and prove it in Section 8.

\section{Definition of 2-microlocal Besov spaces with variable exponents and local mean characterization}

Let $\Omega \subset \mathbb{R}^{n}$. Denote by $\mathcal{P}_{0}(\Omega)$ the set of measurable functions $p(\cdot)$ on $\Omega$ with range in $(0, \infty)$ such that

$$
0<p^{-}=\underset{x \in \Omega}{\operatorname{ess} \inf } p(x), \quad \underset{x \in \Omega}{\operatorname{ess} \sup } p(x)=p^{+}<\infty .
$$

We also denote by $\mathcal{P}(\Omega)$ the set of measurable functions $p(\cdot)$ on $\Omega$ with range in $(1, \infty)$ such that $1<p_{-}$and $p_{+}<\infty$.

For $p \in \mathcal{P}_{0}(\Omega)$, let $L^{p(\cdot)}(\Omega)$ be the set of measurable functions $f$ on $\Omega$ such that for some $\lambda>0$,

$$
\int_{\Omega}\left(\frac{|f(x)|}{\lambda}\right)^{p(x)} \mathrm{d} x \leq 1 .
$$

The infimum of such $\lambda$ is denoted by $\|f\|_{L^{p(\cdot)}(\Omega)}$. The set $L^{p(\cdot)}(\Omega)$ becomes a quasi-Banach function space equipped with the Luxemburg-Nakano norm $\|f\|_{L^{p(\cdot)}(\Omega)}$. More precisely,

$$
\|f\|_{L^{p(\cdot)}(\Omega)}=\inf \left\{\lambda>0: \int_{\Omega}\left(\frac{|f(x)|}{\lambda}\right)^{p(x)} \mathrm{d} x \leq 1\right\} .
$$


When $\Omega=\mathbb{R}^{n}$, we write simply $\|f\|_{L^{p(\cdot)}}:=\|f\|_{L^{p(\cdot)}\left(\mathbb{R}^{n}\right)}$.

To define 2-microlocal Besov spaces with variable exponents, we use the mixed Lebesgue sequence space $\ell^{q(\cdot)}\left(L^{p(\cdot)}\right)$.

Let $p(\cdot), q(\cdot) \in \mathcal{P}_{0}\left(\mathbb{R}^{n}\right)$. The space $\ell^{q(\cdot)}\left(L^{p(\cdot)}\right)$ is the collection of all sequences $\left\{f_{j}\right\}_{j=0}^{\infty}$ of measurable functions on $\mathbb{R}^{n}$ such that

$$
\left\|\left\{f_{j}\right\}_{j=0}^{\infty}\right\|_{\ell^{q(\cdot)}\left(L^{p(\cdot)}\right)}=\inf \left\{\mu>0: \varrho_{\ell^{q(\cdot)}\left(L^{p(\cdot)}\right.}\left(\left\{\frac{f_{j}}{\mu}\right\}_{j=0}^{\infty}\right) \leq 1\right\}<\infty,
$$

where

$$
\varrho_{\ell^{q(\cdot)}\left(L^{p(\cdot)}\right)}\left(\left\{f_{j}\right\}_{j=0}^{\infty}\right)=\sum_{j=0}^{\infty} \inf \left\{\lambda_{j}>0: \int_{\mathbb{R}^{n}}\left(\frac{\left|f_{j}(x)\right|}{\lambda_{j}^{\frac{1}{q(x)}}}\right)^{p(x)} \mathrm{d} x \leq 1\right\} .
$$

Since we assume that $q^{+}<\infty$,

$$
\varrho_{\ell^{q(\cdot)}\left(L^{p(\cdot)}\right)}\left(\left\{f_{j}\right\}_{j=0}^{\infty}\right)=\sum_{j=0}^{\infty}\left\|\left.|| f_{j}\right|^{q(\cdot)}\right\|_{L^{\frac{p(\cdot)}{q(\cdot)}}} .
$$

If $\left\{f_{j}\right\}_{j=M}^{N}$ is a finite sequence of measurable functions on $\mathbb{R}^{n}$, then we define an infinite sequence $\left\{F_{j}\right\}_{j=0}^{\infty}$ of measurable functions on $\mathbb{R}^{n}$,

$$
F_{j}:= \begin{cases}f_{j} & \text { for } j=M, M+1, \ldots, N \\ 0 & \text { otherwise }\end{cases}
$$

and

$$
\left\|\left\{f_{j}\right\}_{j=M}^{N}\right\|_{\ell^{q(\cdot)}\left(L^{p(\cdot)}\right)}:=\left\|\left\{F_{j}\right\}_{j=0}^{\infty}\right\|_{\ell q(\cdot)}\left(L^{p(\cdot))} .\right.
$$

Almeida and Hästö [1] proved that $\|\cdot\|_{\ell q(\cdot)}\left(L^{p(\cdot)}\right)$ is a quasi-norm for all $p(\cdot), q(\cdot) \in$ $\mathcal{P}\left(\mathbb{R}^{n}\right)$ and that $\|\cdot\|_{\ell^{q(\cdot)}\left(L^{p(\cdot)}\right)}$ is a norm when $\frac{1}{p(\cdot)}+\frac{1}{q(\cdot)} \leq 1$. Kempka and Vybíral [9] proved that $\|\cdot\|_{\ell^{q(\cdot)}\left(L^{p(\cdot)}\right)}$ is a norm if $p(\cdot), q(\cdot) \in \mathcal{P}_{0}\left(\mathbb{R}^{n}\right)$ satisfy either $1 \leq q(x) \leq p(x) \leq \infty$ or $\frac{1}{p(x)}+\frac{1}{q(x)} \leq 1$ for almost all $x \in \mathbb{R}^{n}$. Furthermore, they proved that $\|\cdot\|_{\ell^{q(\cdot)}\left(L^{p(\cdot)}\right)}$ does not always become a norm even if $p(\cdot)$ and $q(\cdot)$ satisfy $p^{-}, q^{-} \geq 1$. However, we have the following inequality.

LEMma $1[14$, Lemma 1$]$. Let $p(\cdot), q(\cdot) \in \mathcal{P}_{0}\left(\mathbb{R}^{n}\right)$ and

$$
\alpha=\min \left\{q^{-}, 1\right\} \min \left\{1,\left(\frac{p}{q}\right)^{-}\right\} \text {. }
$$


Then

$$
\left\|\left\{f_{k}+g_{k}\right\}_{k=0}^{\infty}\right\|_{\ell q(\cdot)\left(L^{p(\cdot)}\right)}^{\alpha} \leq\left\|\left\{f_{k}\right\}_{k=0}^{\infty}\right\|_{\ell q^{(\cdot)}\left(L^{p(\cdot)}\right)}^{\alpha}+\left\|\left\{g_{k}\right\}_{k=0}^{\infty}\right\|_{\ell q(\cdot)\left(L^{p(\cdot)}\right)}^{\alpha} .
$$

We denote by $C^{\log }\left(\mathbb{R}^{n}\right)$ the set of all real valued functions $p(\cdot): \mathbb{R}^{n} \rightarrow \mathbb{R}$ satisfying following conditions: There exist constants $C_{\log }(p)$ and $p_{\infty} \in \mathbb{R}$ such that

$$
|p(x)-p(y)| \leq \frac{C_{\log }(p)}{\log \left(e+|x-y|^{-1}\right)} \quad\left(x, y \in \mathbb{R}^{n}, x \neq y\right)
$$

and

$$
\left|p(x)-p_{\infty}\right| \leq \frac{C_{\log }(p)}{\log (e+|x|)} \quad\left(x \in \mathbb{R}^{n}\right) .
$$

DEFINITION 1 (Resolution of unity). The set $\Phi\left(\mathbb{R}^{n}\right)$ is the collection of all systems $\left\{\varphi_{j}\right\}_{j=0}^{\infty} \subset \mathcal{S}\left(\mathbb{R}^{n}\right)$ satisfying the following conditions:

(i) $\varphi_{0}(x)=1$ for $|x| \leq 1$,

(ii) $\operatorname{supp} \varphi_{0} \subset\left\{x \in \mathbb{R}^{n}:|x| \leq 2\right\}$,

(iii) $\varphi_{j}(x)=\varphi_{0}\left(2^{-j} x\right)-\varphi_{0}\left(2^{-j+1} x\right)$ for $j \in \mathbb{N}$.

REMARK 1. Let $\left\{\varphi_{j}\right\}_{j=0}^{\infty} \in \Phi\left(\mathbb{R}^{n}\right)$. Then one may verify easily that

$$
\sum_{j=0}^{\infty} \varphi_{j}(x)=1
$$

for all $x \in \mathbb{R}^{n}$.

DEFINITION 2 [18, Admissible weight sequence]. Let $\alpha \geq 0$ and $\alpha_{1}, \alpha_{2} \in \mathbb{R}$ with $\alpha_{1} \leq \alpha_{2}$. A sequence of non-negative measurable weight functions $\boldsymbol{w}=\left\{w_{j}\right\}_{j=0}^{\infty}$ belongs to the class $\mathcal{W}_{\alpha_{1}, \alpha_{2}}^{\alpha}\left(\mathbb{R}^{n}\right)$ if the following hold.

(i) There exists a constant $C>0$ such that $0<w_{j}(x) \leq C w_{j}(y)\left(1+2^{j}|x-y|\right)^{\alpha}$ for all $j \in \mathbb{N}_{0}$ and all $x, y \in \mathbb{R}^{n}$.

(ii) For all $j \in \mathbb{N}_{0}$ we have $2^{\alpha_{1}} w_{j}(x) \leq w_{j+1}(x) \leq 2^{\alpha_{2}} w_{j}(x)$ for all $x \in \mathbb{R}^{n}$.

Such a system $\left\{w_{j}\right\}_{j=0}^{\infty} \in \mathcal{W}_{\alpha_{1}, \alpha_{2}}^{\alpha}\left(\mathbb{R}^{n}\right)$ is called an admissible weight sequence.

REMARK 2. Let $s(\cdot) \in C^{\log }\left(\mathbb{R}^{n}\right)$. Then $\left\{2^{j s(\cdot)}\right\}_{j=0}^{\infty}$ is an admissible weight sequence with $\alpha_{1}=s^{-}, \alpha_{2}=s^{+}$and $\alpha>C_{\log }(s)$ (see Lemma 12).

Another fundamental example of an admissible weight sequence is a 2-microlocal weights. For a fixed nonempty set $U \subset \mathbb{R}^{n}$ and $s^{\prime} \in \mathbb{R}$, We denote $\operatorname{dist}(x, U)=\inf _{z \in U}|x-z|$. The 2-microlocal weights $\left\{w_{j}\right\}_{j=0}^{\infty}$ is given by

$$
w_{j}(x)=\left(1+2^{j} \operatorname{dist}(x, U)\right)^{s^{\prime}}
$$


for any $j \in \mathbb{N}_{0}$. This 2-microlocal weights $\left\{w_{j}\right\}_{j=0}^{\infty}$ is an admissible weight sequence with $\alpha_{1}=\min \left(0, s^{\prime}\right), \alpha_{2}=\max \left(0, s^{\prime}\right)$ and $\alpha=\left|s^{\prime}\right|$.

Further examples are given in [6].

Let $\phi$ be a continuous function on $\mathbb{R}^{n}$ or a sum of finitely many characteristic functions of cubes in $\mathbb{R}^{n}$. Then $\phi(D)$ is defined by $\phi(D) f=\mathcal{F}^{-1}[\phi \cdot \mathcal{F} f]$.

We introduce the 2-microlocal Besov spaces with variable exponents defined by Kempka and Vybíral [10].

DEFINITION 3 (2-microlocal Besov spaces with variable exponents). Let $p(\cdot), q(\cdot) \in C^{\log }\left(\mathbb{R}^{n}\right) \cap \mathcal{P}_{0}\left(\mathbb{R}^{n}\right),\left\{\varphi_{j}\right\}_{j=0}^{\infty} \in \Phi\left(\mathbb{R}^{n}\right)$ and $\boldsymbol{w}=\left\{w_{j}\right\}_{j=0}^{\infty} \in \mathcal{W}_{\alpha_{1}, \alpha_{2}}^{\alpha}\left(\mathbb{R}^{n}\right)$. Then we define

$$
B_{p(\cdot), q(\cdot)}^{\boldsymbol{w}}\left(\mathbb{R}^{n}\right)=\left\{f \in \mathcal{S}^{\prime}\left(\mathbb{R}^{n}\right):\|f\|_{B_{p(\cdot), q(\cdot)}^{\boldsymbol{w}}}<\infty\right\}
$$

where

$$
\|f\|_{B_{p(\cdot), q(\cdot)}^{w}}^{w}=\inf \left\{\lambda>0: \sum_{j=0}^{\infty}\left\|\left(\frac{\left|w_{j} \varphi_{j}(D) f\right|}{\lambda}\right)^{q(\cdot)}\right\|_{L^{p(\cdot) / q(\cdot)}} \leq 1\right\} .
$$

Kempka and Vybíral proved the norm of 2-microlocal Besov spaces with variable exponents does not depend on the resolution of unity (Definition 1) by the local mean characterization (Theorem 1).

Let $\left\{\psi_{k}\right\}_{k=0}^{\infty} \subset \mathcal{S}\left(\mathbb{R}^{n}\right), f \in \mathcal{S}^{\prime}\left(\mathbb{R}^{n}\right)$ and $a>0$. Then the Peetre maximal operator is defined by

$$
\left(\psi_{k}^{*} f\right)_{a}(x)=\sup _{y \in \mathbb{R}^{n}} \frac{\left|\left(\psi_{k} * f\right)(y)\right|}{\left(1+\left|2^{k}(y-x)\right|\right)^{a}},
$$

for $k \in \mathbb{N}_{0}$ and $x \in \mathbb{R}^{n}$.

In order to state local mean characterization, let us consider $\kappa_{0}, \kappa \in \mathcal{S}\left(\mathbb{R}^{n}\right)$ such that

$$
\left|\mathcal{F} \kappa_{0}(\xi)\right|>0
$$

on $\left\{x \in \mathbb{R}^{n}:|\xi|<2 \varepsilon\right\}$ and

$$
\left|\mathcal{F}_{\kappa}(\xi)\right|>0
$$

on $\left\{x \in \mathbb{R}^{n}: \varepsilon / 2<|\xi|<2 \varepsilon\right\}$ for some $\varepsilon>0$. Here (4) and (5) are called the Tauberian conditions. Furthermore we assume the moment condition on $\kappa$, that is,

$$
\int_{\mathbb{R}^{n}} x^{\beta} \kappa(x) \mathrm{d} x=0
$$

for any $0 \leq|\beta|<S$, where $S \in \mathbb{N}_{0}$. If $S=0$, there are no moment condition (6) on $\kappa$. 
We put

$$
\kappa_{j}(x)=2^{j n} \kappa\left(2^{j} x\right)
$$

for $j \in \mathbb{N}_{0}$.

THEOREM 1 (Local mean characterization [10, Theorem 6]).

Let $\boldsymbol{w}=\left\{w_{j}\right\}_{j=0}^{\infty} \in \mathcal{W}_{\alpha_{1}, \alpha_{2}}^{\alpha}\left(\mathbb{R}^{n}\right), p(\cdot), q(\cdot) \in C^{\log }\left(\mathbb{R}^{n}\right) \cap \mathcal{P}_{0}\left(\mathbb{R}^{n}\right)$. Furthermore, let a $\in \mathbb{R}$ and $S \in \mathbb{N}_{0}$ satisfy $a>\frac{n+C_{\log }(1 / q)}{p^{-}}+\alpha$ and $S>\alpha_{2}$. Then we have

$$
\|f\|_{B_{p(\cdot), q(\cdot)}^{w}} \sim\left\|\left\{\left(\kappa_{k} * f\right) w_{k}\right\}_{k=0}^{\infty}\right\|_{\ell q(\cdot)}\left(L^{p(\cdot)}\right) \sim\left\|\left\{w_{k}(\cdot)\left(\kappa_{k}^{*} f\right)_{a}(\cdot)\right\}_{k=0}^{\infty}\right\|_{\ell q(\cdot)}\left(L^{p(\cdot)}\right)
$$

for all $f \in \mathcal{S}^{\prime}\left(\mathbb{R}^{n}\right)$.

\section{Fundamental results}

As well as classical analysis, the following generalized Hölder's inequality holds.

THEOREM 2 [11, Theorem 2.1]. Let $p(\cdot): \mathbb{R}^{n} \rightarrow[1, \infty]$ be a measurable function and let $p^{\prime}(\cdot): \mathbb{R}^{n} \rightarrow[1, \infty]$ be defined as $\frac{1}{p(\cdot)}+\frac{1}{p^{\prime}(\cdot)}=1$. Then generalized Hölder's inequality

$$
\int_{\mathbb{R}^{n}}|f(x) g(x)| \mathrm{d} x \leq\left(1+\frac{1}{p_{-}}-\frac{1}{p_{+}}\right)\|f\|_{L^{p(\cdot)}}\|g\|_{L^{p^{\prime}(\cdot)}}
$$

holds for every $f \in L^{p(\cdot)}\left(\mathbb{R}^{n}\right)$ and $g \in L^{p^{\prime}(\cdot)}\left(\mathbb{R}^{n}\right)$.

Lemma 2. Let $p(\cdot), q(\cdot) \in \mathcal{P}_{0}\left(\mathbb{R}^{n}\right)$ and $f \in L^{p(\cdot)}\left(\mathbb{R}^{n}\right)$. Then

$$
\min \left\{\|f\|_{L^{p(\cdot)}}^{q^{-}},\|f\|_{L^{p(\cdot)}}^{q^{+}}\right\} \leq\left\|f^{q(\cdot)}\right\|_{L^{p(\cdot) / q(\cdot)}} \leq \max \left\{\|f\|_{L^{p(\cdot)}}^{q^{-}},\|f\|_{L^{p(\cdot)}}^{q^{+}}\right\}
$$

holds.

PROOF. When $\|f\|_{L^{p(\cdot)}}=0$, it is obvious that $\left\|f^{q(\cdot)}\right\|_{L^{p(\cdot) / q(\cdot)}}=0$. Hence we consider the case that $\|f\|_{L^{p(\cdot)}} \neq 0$. We normalize $f$ by putting $g=f /\|f\|_{L^{p(\cdot)}}$. Then $\|g\|_{L^{p(\cdot)}}=1$. Therefore, we see that

$$
1=\int_{\mathbb{R}^{n}}\left(\frac{|g(x)|}{1}\right)^{p(x)} \mathrm{d} x=\int_{\mathbb{R}^{n}}\left(\frac{\left|g(x)^{q(x)}\right|}{1}\right)^{p(x) / q(x)} \mathrm{d} x
$$

holds. This implies that $\left\|\left(\frac{f}{\|f\|_{L^{p(\cdot)}}}\right)^{q(\cdot)}\right\|_{L^{p(\cdot) / q(\cdot)}}=\left\|g^{q(\cdot)}\right\|_{L^{p(\cdot) / q(\cdot)}}=1$. If $\|f\|_{L^{p(\cdot)}}>1$, then it is easy to see that

$$
\left\|\frac{f^{q(\cdot)}}{\|f\|_{L^{p^{(\cdot)}}}^{q^{-}}}\right\|_{L^{p(\cdot) / q(\cdot)}} \leq\left\|\left(\frac{f}{\|f\|_{L^{p(\cdot)}}}\right)^{q(\cdot)}\right\|_{L^{p(\cdot) / q(\cdot)}}=1 \leq\left\|\frac{f^{q(\cdot)}}{\|f\|_{L^{p(\cdot)}}^{q^{+}}}\right\|_{L^{p(\cdot) / q(\cdot)}} .
$$


Hence we obtain

$$
\|f\|_{L^{p(\cdot)}}^{q^{+}} \leq\left\|f^{q(\cdot)}\right\|_{L^{p(\cdot) / q(\cdot)}} \leq\|f\|_{L^{p(\cdot)}}^{q^{-}} .
$$

An argument similar to above shows the case that $\|f\|_{L^{p(\cdot)}}<1$.

Almeida and Hästö [1] showed that the Feffermann-Stein maximal inequality does not hold if $q(\cdot)$ is not a constant function. However, Almeida and Hästö [1] got a helpful inequality (Theorem 3), which takes the place of the Feffermann-Stein maximal inequality.

Let

$$
\eta_{l}(x)=(1+|x|)^{-l} \quad \text { and } \quad \eta_{v, l}(x)=2^{v n} \eta_{l}\left(2^{v} x\right)
$$

for $v \in \mathbb{N}_{0}$ and a positive real number $l$. Almeida and Hästö [1] showed the following helpful theorem for the $\ell^{q(\cdot)}\left(L^{p(\cdot)}\right)$ quasi-norm.

THEOREM 3 [1, Lemma 4.7]. Let $p(\cdot), q(\cdot) \in C^{\log }\left(\mathbb{R}^{n}\right)$ with $1<p^{-} \leq p^{+}<\infty$ and $1<q^{-} \leq q^{+}<\infty$. Then the inequality

$$
\left\|\left\{\eta_{k, l} * f_{k}\right\}_{k=0}^{\infty}\right\|_{\ell q(\cdot)\left(L^{p(\cdot)}\right)} \lesssim\left\|\left\{f_{k}\right\}_{k=0}^{\infty}\right\|_{\ell q(\cdot)}\left(L^{p(\cdot)}\right)
$$

holds for every sequence $\left\{f_{k}\right\}_{k=0}^{\infty}$ of $L_{\text {loc }}^{1}$-functions and $l>2 n$.

The next lemma is called "the $r$-trick".

Lemma 3 [3, Lemma A.7]. Let $r>0, v \in \mathbb{N}_{0}$ and $l>n$. Then

$$
|f(x)| \lesssim\left(\eta_{\nu, l} *|f|^{r}(x)\right)^{\frac{1}{r}}
$$

holds for all $x \in \mathbb{R}^{n}$ and $f \in \mathcal{S}^{\prime}\left(\mathbb{R}^{n}\right)$ with $\operatorname{supp} \mathcal{F} f \subset\left\{\xi:|\xi| \leq 2^{v+1}\right\}$, where the implicit positive constant depends only on $r, l$ and $n$.

\section{Atomic decomposition}

In the case where the summability parameter is constant, Kempka [8] got an atomic decomposition for $B_{p(\cdot), q}^{\boldsymbol{w}}\left(\mathbb{R}^{n}\right)$. If $s(\cdot) \in C^{\log }\left(\mathbb{R}^{n}\right) \cap L^{\infty}\left(\mathbb{R}^{n}\right)$ and $w_{j}(x)=2^{j s(x)}$, then the space $B_{p(\cdot), q(\cdot)}^{\boldsymbol{w}}\left(\mathbb{R}^{n}\right)$ coincides with the Besov space with variable exponents $B_{p(\cdot), q(\cdot)}^{s(\cdot)}\left(\mathbb{R}^{n}\right)$. The atomic decomposition for $B_{p(\cdot), q(\cdot)}^{s(\cdot)}\left(\mathbb{R}^{n}\right)$ was studied by Drihem [4]. Therefore, we combine the argument of [8] with [4] to prove an atomic decomposition theorem (Theorem 4).

Definition 4 (atom). Let $K, L \in \mathbb{N}_{0}$. A function $a \in C^{k}\left(\mathbb{R}^{n}\right)$ is called a $[K, L]$ atom centered at $Q_{v m}, v \in \mathbb{N}_{0}$ and $m \in \mathbb{Z}^{n}$, if

$$
\begin{aligned}
& \text { supp } a \subset 3 Q_{\nu m}, \\
& \left|D^{\beta} a(x)\right| \leq 2^{|\beta| v},
\end{aligned}
$$


for $0 \leq|\beta| \leq K$ and if

$$
\int_{\mathbb{R}^{n}} x^{\beta} a(x) \mathrm{d} x=0
$$

for $0 \leq|\beta|<L$ and $v \geq 1$.

The condition (10) is called the moment condition. In the case where $a$ is an atom centered at $Q_{0 m}$ or the case $L=0$, the moment condition (10) is not required.

Definition 5 (Coefficients space). Let $\boldsymbol{w}=\left\{w_{j}\right\}_{j=0}^{\infty} \in \mathcal{W}_{\alpha_{1}, \alpha_{2}}^{\alpha}\left(\mathbb{R}^{n}\right)$ and $p(\cdot), q(\cdot) \in C^{\log }\left(\mathbb{R}^{n}\right) \cap \mathcal{P}_{0}\left(\mathbb{R}^{n}\right)$. Then for all complex valued sequences $\lambda=\left\{\lambda_{v m} \in \mathbb{C}:\right.$ $\left.v \in \mathbb{N}_{0}, m \in \mathbb{Z}^{n}\right\}$ we define

$$
b_{p(\cdot), q(\cdot)}^{\boldsymbol{w}}\left(\mathbb{R}^{n}\right)=\left\{\lambda:\|\lambda\|_{b_{p(\cdot), q(\cdot)}^{w}}<\infty\right\}
$$

where

$$
\|\lambda\|_{b_{p(\cdot), q(\cdot)}^{w}}=\left\|\left\{\sum_{m \in \mathbb{Z}^{n}}\left|\lambda_{\nu m}\right| w_{\nu}\left(2^{-v} m\right) \chi_{\nu m}(\cdot)\right\}_{\nu=0}^{\infty}\right\|_{\ell q(\cdot)\left(L^{p(\cdot)}\right)} .
$$

When the summability parameter is $\infty$, we define

$$
b_{p(\cdot), \infty}^{\boldsymbol{w}}\left(\mathbb{R}^{n}\right)=\left\{\lambda:\|\lambda\|_{p(\cdot), \infty}^{\boldsymbol{w}}<\infty\right\}
$$

where

$$
\|\lambda\|_{b_{p(\cdot), \infty}^{w}}=\inf _{\nu \in \mathbb{N}_{0}}\left\{\left\|\sum_{m \in \mathbb{Z}^{n}}\left|\lambda_{v m}\right| w_{\nu}\left(2^{-v} m\right) \chi_{\nu m}(\cdot)\right\|_{L^{p(\cdot)}}\right\} .
$$

THEOREM 4 (Atomic decomposition theorem). Let $\boldsymbol{w}=\left\{w_{j}\right\}_{j=0}^{\infty} \in \mathcal{W}_{\alpha_{1}, \alpha_{2}}^{\alpha}\left(\mathbb{R}^{n}\right)$ and $p(\cdot), q(\cdot) \in C^{\log }\left(\mathbb{R}^{n}\right) \cap \mathcal{P}_{0}\left(\mathbb{R}^{n}\right)$. Additionally, let $K, L \in \mathbb{N}_{0}$ satisfy $K>\alpha_{2}$ and $L>$ $n\left(\frac{1}{\min \left\{1, p^{-}\right\}}-1\right)-\alpha_{1}$, then we have the following:

(a) For each $f \in B_{p(\cdot), q(\cdot)}^{\boldsymbol{w}}\left(\mathbb{R}^{n}\right)$ there exist $\lambda \in b_{p(\cdot), q(\cdot)}^{\boldsymbol{w}}\left(\mathbb{R}^{n}\right)$ and $[K, L]$-atoms

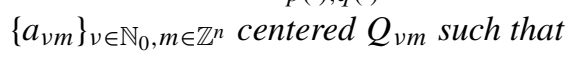

$$
f=\sum_{\nu=0}^{\infty} \sum_{m \in \mathbb{Z}^{n}} \lambda_{\nu m} a_{\nu m}
$$

holds in the sense of $\mathcal{S}^{\prime}\left(\mathbb{R}^{n}\right)$. Moreover

$$
\|\lambda\|_{b_{p(\cdot), q(\cdot)}^{w}} \lesssim\|f\|_{B_{p(\cdot), q(\cdot)}^{w}}
$$

holds, where the implicit positive constant is universal for all $f \in B_{p(\cdot), q(\cdot)}^{w}\left(\mathbb{R}^{n}\right)$. 


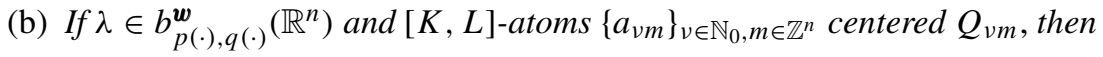

$$
f=\sum_{\nu=0}^{\infty} \sum_{m \in \mathbb{Z}^{n}} \lambda_{\nu m} a_{\nu m}
$$

in the sense of $\mathcal{S}^{\prime}\left(\mathbb{R}^{n}\right)$, belongs to the space $B_{p(\cdot), q(\cdot)}^{w}\left(\mathbb{R}^{n}\right)$. Moreover,

$$
\|f\|_{B_{p(\cdot), q(\cdot)}^{w}} \lesssim\|\lambda\|_{b_{p(\cdot), q(\cdot)}^{w}}
$$

holds, where the implicit positive constant is universal for all $\lambda \in b_{p(\cdot), q(\cdot)}^{\boldsymbol{w}}\left(\mathbb{R}^{n}\right)$.

To prove Theorem 4 we need the following lemmas.

Lemma 4 [5, Lemma 3.3]. Let $\left\{\varphi_{j}\right\}_{j=0}^{\infty}$ be a resolution of unity and let $a_{v m}$ be an $[K, L]$-atom. Then

$$
\left|\mathcal{F}^{-1} \varphi_{j} * a_{v m}(x)\right| \lesssim \begin{cases}2^{(v-j) K}\left(1+2^{j}\left|x-2^{-v} m\right|\right)^{-M} & \text { if } v \leq j \\ 2^{(j-v)(L+n)}\left(1+2^{j}\left|x-2^{-v} m\right|\right)^{-M} & \text { if } j \leq v,\end{cases}
$$

where $M$ is a sufficiently large number.

The next lemma is a direct corollary of [14, Lemma 4.13].

Lemma 5. Let $0<t<1, j, v \in \mathbb{N}_{0}$ and $\left\{\lambda_{v m}\right\}_{v \in \mathbb{N}_{0}, m \in \mathbb{Z}^{n}}$ be a family of positive numbers. Furthermore let $M>0$ be sufficiently large. Then

$$
\begin{aligned}
& \sum_{m \in \mathbb{Z}^{n}} \lambda_{v m}\left(1+2^{j}\left|x-2^{-v} m\right|\right)^{-M} \\
& \lesssim 2^{h_{n} \alpha} \max \left(1,2^{(\nu-j) n / t}\right)\left(\left[\eta_{v, \alpha t} *\left(\sum_{m \in \mathbb{Z}^{n}} \lambda_{v m}^{t} \chi_{v m}(\cdot)\right)\right](x)\right)^{1 / t}
\end{aligned}
$$

holds for any $x \in \mathbb{R}^{n}$ and for any positive real number $\alpha>0$, where $h_{n}$ is a positive number depending only on $n$.

Lemma 6 [4, Lemma 3]. Let $0<a<1,0<q \leq \infty$ and $\delta>0$ and let $\left\{\varepsilon_{k}\right\}_{k=0}^{\infty}$ be a sequence of positive real numbers, such that

$$
I=\left\|\left\{\varepsilon_{k}\right\}_{k=0}^{\infty}\right\|_{\ell q}<\infty .
$$

The sequence $\left\{\delta_{k}: \delta_{k}=\sum_{j=0}^{\infty} a^{|k-j| \delta} \varepsilon_{j}\right\}_{k=0}^{\infty}$ is in $\ell^{q}$ with

$$
\left\|\left\{\delta_{k}\right\}_{k=0}^{\infty}\right\|_{\ell q} \lesssim I
$$

where the implicit positive constant depends only on a and $q$.

Next lemma is a Hardy type inequality in the variable exponents settings. In the case where $q(\cdot)$ is constant, Lemma 7 above was proved by $\mathrm{Xu}[16$, Lemma 3]. 
LEMmA 7. Let $p(\cdot), q(\cdot) \in \mathcal{P}_{0}\left(\mathbb{R}^{n}\right)$. For any sequence $\left\{g_{j}\right\}_{j=0}^{\infty}$ of nonnegative measurable functions on $\mathbb{R}^{n}$ and $\delta>0$ let

$$
G_{j}(x)=\sum_{k=0}^{\infty} 2^{-|k-j| \delta} g_{k}(x)
$$

for all $x \in \mathbb{R}^{n}$ and $j \in \mathbb{N}_{0}$. Then

$$
\left\|\left\{G_{j}\right\}_{j=0}^{\infty}\right\|_{\ell^{q(\cdot)}\left(L^{p(\cdot)}\right)} \lesssim\left\|\left\{g_{j}\right\}_{j=0}^{\infty}\right\|_{\ell^{q(\cdot)}\left(L^{p(\cdot)}\right)}
$$

holds, where the implicit positive constant depends only on $p(\cdot), q(\cdot)$ and $\delta$.

PROOF. Let $0<q<\infty$. We recall the following inequality which can be proved by using an argument similar to the proof of Jensen's inequality,

$$
\left(\sum_{k=0}^{\infty} 2^{-k \delta} a_{k}\right)^{q} \lesssim \sum_{k=0}^{\infty} 2^{-k(\delta-\varepsilon) q} a_{k}^{q}
$$

holds for any $\varepsilon<\delta$, where the implicit positive constant depends only on $\delta$ and $q$. Therefore, there exist a constant $\delta_{1}>0$ which depend only on $\delta$ such that

$$
\left(\sum_{k=0}^{\infty} 2^{-|k-j| \delta} g_{k}(x)\right)^{q(x)} \lesssim \sum_{k=0}^{\infty}\left(2^{-|k-j| \delta_{1}} g_{k}(x)\right)^{q(x)}
$$

for any $j \in \mathbb{N}_{0}$.

Let $r>0$ satisfy $r(p(\cdot) / q(\cdot))^{-}>1$. Then by using same argument as above, there exist a constant $\delta_{2}>0$ which depend only on $\delta$ such that

$$
\begin{aligned}
\left\|\left(\frac{\sum_{k=0}^{\infty} 2^{-|k-j| \delta} g_{k}(\cdot)}{\lambda}\right)^{q(\cdot)}\right\|_{L^{p(\cdot) / q(\cdot)}} & =\left\|\left(\frac{\sum_{k=0}^{\infty} 2^{-|k-j| \delta} g_{k}(\cdot)}{\lambda}\right)^{q(\cdot) / r}\right\|_{L^{r p(\cdot) / q(\cdot)}}^{r} \\
& \lesssim\left\|\sum_{k=0}^{\infty} 2^{-|k-j| \delta_{1} q(\cdot) / r}\left(\frac{g_{k}(\cdot)}{\lambda}\right)^{q(\cdot) / r}\right\|_{L^{r p(\cdot) / q(\cdot)}}^{r}
\end{aligned}
$$

holds. By the triangle inequality and the similar argument as above, we have

$$
\begin{aligned}
\left\|\left(\frac{\sum_{k=0}^{\infty} 2^{-|k-j| \delta} g_{k}(\cdot)}{\lambda}\right)^{q(\cdot)}\right\|_{L^{p(\cdot) / q(\cdot)}} & \lesssim\left(\sum_{k=0}^{\infty} 2^{-|k-j| \delta_{1} q^{-} / r}\left\|\left(\frac{g_{k}(\cdot)}{\lambda}\right)^{q(\cdot) / r}\right\|_{L^{r p(\cdot) / q(\cdot)}}\right)^{r} \\
& \lesssim \sum_{k=0}^{\infty} 2^{-|k-j| \delta_{2} q^{-}}\left\|\left(\frac{g_{k}(\cdot)}{\lambda}\right)^{q(\cdot)}\right\|_{L^{p(\cdot) / q(\cdot)}}
\end{aligned}
$$

Hence, by (16) and Lemma 6, we see that

$$
\sum_{j=0}^{\infty}\left\|\left(\frac{G_{j}(\cdot)}{\lambda}\right)^{q(\cdot)}\right\|_{L^{p(\cdot) / q(\cdot)}}=\sum_{j=0}^{\infty}\left\|\left(\frac{\sum_{k=0}^{\infty} 2^{-|k-j| \delta} g_{k}(\cdot)}{\lambda}\right)^{q(\cdot)}\right\|_{L^{p(\cdot) / q(\cdot)}}
$$




$$
\begin{aligned}
& \lesssim \sum_{j=0}^{\infty} \sum_{k=0}^{\infty} 2^{-|k-j| \delta_{2} q^{-}}\left\|\left(\frac{g_{k}(\cdot)}{\lambda}\right)^{q(\cdot)}\right\|_{L^{p(\cdot) / q(\cdot)}} \\
& \lesssim \sum_{k=0}^{\infty}\left\|\left(\frac{g_{k}(\cdot)}{\lambda}\right)^{q(\cdot)}\right\|_{L^{p(\cdot) / q(\cdot)}} \sim 1,
\end{aligned}
$$

where we choose $\lambda=\left\|\left\{g_{j}\right\}_{j=0}^{\infty}\right\|_{\ell q(\cdot)\left(L^{p(\cdot)}\right)}$.

This inequality implies (15).

We also need a partition of unity of Calderón type.

LEMmA 8 (Partition of unity of Calderón type [5, Theorem 6]). Let $\left\{\varphi_{j}\right\}_{j=0}^{\infty} \in$ $\Phi\left(\mathbb{R}^{n}\right)$ be a resolution of unity and $R \in \mathbb{N}$. Then there exist functions $\theta_{0}, \theta \in \mathcal{S}\left(\mathbb{R}^{n}\right)$ such that

$$
\begin{array}{r}
\operatorname{supp} \theta_{0}, \operatorname{supp} \theta \subset\{x:|x| \leq 1\}, \\
\left|\mathcal{F} \theta_{0}(\xi)\right| \geq c_{0}>0 \text { for }|\xi| \leq 2, \\
|\mathcal{F} \theta(\xi)| \geq c>0 \text { for } \frac{1}{2} \leq|\xi| \leq 2 \\
\int_{\mathbb{R}^{n}} x^{\gamma} \theta(x) \mathrm{d} x=0 \text { for } 0 \leq|\gamma| \leq R
\end{array}
$$

and

$$
\mathcal{F} \theta_{0}(\xi) \mathcal{F} \psi_{0}(\xi)+\sum_{j=1}^{\infty} \mathcal{F} \theta\left(2^{-j} \xi\right) \mathcal{F} \psi\left(2^{-j} \xi\right)=1
$$

for all $\xi \in \mathbb{R}^{n}$, where the functions $\psi_{0}, \psi \in \mathcal{S}\left(\mathbb{R}^{n}\right)$ are defined by

$$
\mathcal{F} \psi_{0}(\xi)=\frac{\varphi_{0}(\xi)}{\mathcal{F} \theta_{0}(\xi)} \quad \text { and } \quad \mathcal{F} \psi(\xi)=\frac{\varphi_{1}(2 \xi)}{\mathcal{F} \theta(\xi)} .
$$

Proof of Theorem 4. We use an argument similar to [8, Theorem 3.12, Theorem 3.13].

Step 1. Kempka [8, Proof of Theorem 3.12] showed $f \in \mathcal{S}^{\prime}\left(\mathbb{R}^{n}\right)$ can be written by $f(x)=\sum_{m \in \mathbb{Z}^{n}} \int_{Q_{0 m}} \theta_{0}(x-y)\left(\psi_{0} * f\right)(y) \mathrm{d} y+\sum_{\nu=1}^{\infty} \sum_{m \in \mathbb{Z}^{n}} 2^{v m} \int_{Q_{v m}} \theta\left(2^{v}(x-y)\right)\left(\psi_{v} * f\right)(y) \mathrm{d} y$, where $\theta, \psi_{0}$ and $\psi$ as in Lemma 8 and $\psi_{v}=2^{v n} \psi\left(2^{\nu} \cdot\right)$. For $v \in \mathbb{N}$ and $m \in \mathbb{Z}^{n}$, each coefficient $\lambda$ is defined by

$$
\lambda_{v m}=C_{\theta} \sup _{y \in Q_{v m}}\left|\left(\psi_{k} * f\right)(y)\right|,
$$


where $C_{\theta}=\max \left\{\sup _{|x| \leq 1}\left|D^{\beta} \theta(x)\right|:|\beta|<K\right\}$. Note that $\lambda_{v m} \geq 0$ for any $v \in \mathbb{N}_{0}$ and $m \in \mathbb{Z}^{n}$. We define a function $a_{v m}(x)$ by

$$
a_{v m}(x)= \begin{cases}\frac{2^{v m}}{\lambda_{v m}} \int_{Q_{v m}} \theta\left(2^{v}(x-y)\right)\left(\psi_{v} * f\right)(y) \mathrm{d} y & \left(\lambda_{v m} \neq 0\right) \\ 0 & \left(\lambda_{v m}=0\right) .\end{cases}
$$

Then (11) holds and the properties of $\theta_{0}, \psi_{0}, \theta$ and $\psi_{\nu}$ imply that $a_{v m}$ satisfies the conditions of $[K, L]$-atoms centered at $Q_{v m}$.

For each fixed $v \in \mathbb{N}_{0}$ and $a>\frac{n}{\min \left\{1, p^{-}, q^{-}\right\}}+\alpha$, Kempka [8, Proof of Theorem 3.12] proved

$$
\sum_{m \in \mathbb{Z}^{n}} w_{v}(x) \lambda_{v m} \chi_{v m}(x) \lesssim w_{v}(x)\left(\psi_{v}^{*} f\right)_{a}(x) .
$$

By the property of admissible weight sequence (Definition 2) and $2^{v}\left|x-2^{-v} m\right| \lesssim 1$ for any $x \in Q_{v m}$, we have

$$
\sum_{m \in \mathbb{Z}^{n}} w_{v}\left(2^{-v} m\right) \lambda_{v m} \chi_{v m}(x) \lesssim \sum_{m \in \mathbb{Z}^{n}} w_{v}(x) \lambda_{v m} \chi_{v m}(x) \lesssim w_{v}(x)\left(\psi_{v}^{*} f\right)_{a}(x) .
$$

Since $\psi_{0}, \psi \in \mathcal{S}\left(\mathbb{R}^{n}\right)$ satisfy the Tauberian conditions (4), (5) and the moment condition (6), we can use Theorem 1 . Hence we see that

$$
\begin{aligned}
\|\lambda\|_{b_{p(\cdot), q(\cdot)}^{w}}^{w} & =\left\|\left\{\sum_{m \in \mathbb{Z}^{n}} w_{\nu}\left(2^{-v} m\right) \lambda_{v m} \chi_{\nu m}(\cdot)\right\}_{\nu=0}^{\infty}\right\| \|_{\ell q(\cdot)\left(L^{p(\cdot)}\right)} \\
& \lesssim\left\|\left\{w_{\nu}(\cdot)\left(\psi_{\nu}^{*} f\right)_{a}(\cdot)\right\}_{\nu=0}^{\infty}\right\|_{\ell^{q(\cdot)}\left(L^{p(\cdot)}\right)} \lesssim\|f\|_{B_{p(\cdot), q(\cdot)}^{w}} .
\end{aligned}
$$

Step 2. Let $f=\sum_{\nu=0}^{\infty} \sum_{m \in \mathbb{Z}^{n}} \lambda_{\nu m} a_{\nu m}$ as in (13). Then we divide $w_{j} \varphi_{j}(D) f$ into two parts:

$$
\begin{aligned}
w_{j} \varphi_{j}(D) f & =\sum_{\nu=0}^{\infty} \sum_{m \in \mathbb{Z}^{n}} w_{j} \lambda_{\nu m} \varphi_{j}(D) a_{\nu m} \\
& =\sum_{\nu=0}^{j} \sum_{m \in \mathbb{Z}^{n}} w_{j} \lambda_{\nu m} \varphi_{j}(D) a_{\nu m}+\sum_{\nu=j+1}^{\infty} \sum_{m \in \mathbb{Z}^{n}} w_{j} \lambda_{v m} \varphi_{j}(D) a_{\nu m} \\
& =: f_{j}+f^{j}
\end{aligned}
$$

Then we have

$$
\begin{aligned}
\|f\|_{B_{p(\cdot), q(\cdot)}^{w}}^{w} & =\left\|\left\{w_{j}(\cdot) \varphi_{j}(D) f\right\}_{j=0}^{\infty}\right\|_{\ell^{q(\cdot)}\left(L^{p(\cdot)}\right)} \\
& \lesssim\left\|\left\{f_{j}\right\}_{j=0}^{\infty}\right\|_{\ell^{q(\cdot)}\left(L^{p(\cdot)}\right)}+\left\|\left\{f^{j}\right\}_{j=0}^{\infty}\right\|_{\ell q(\cdot)\left(L^{p(\cdot)}\right)} .
\end{aligned}
$$


Firstly, we consider

$$
\left\|\left\{f_{j}\right\}_{j=0}^{\infty}\right\|_{\ell q(\cdot)\left(L^{p(\cdot)}\right)}=\left\|\left\{\sum_{\nu=0}^{j} \sum_{m \in \mathbb{Z}^{n}} \lambda_{v m} w_{j}(\cdot) \varphi_{j}(D) a_{\nu m}(\cdot)\right\}_{j=0}^{\infty}\right\| \|_{\ell q(\cdot)\left(L^{p(\cdot)}\right)} .
$$

By Lemma 4 and the property of admissible weight sequence (Definition 2), we have

$$
\begin{aligned}
\mid \sum_{\nu=0}^{j} & \sum_{m \in \mathbb{Z}^{n}} \lambda_{\nu m} w_{j}(x) \varphi_{j}(D) a_{\nu m}(x) \mid \\
& \lesssim \sum_{\nu=0}^{j} \sum_{m \in \mathbb{Z}^{n}} 2^{(\nu-j) K}\left|\lambda_{v m}\right| w_{j}(x)\left(1+2^{j}\left|x-2^{-v} m\right|\right)^{-M} \\
& \lesssim \sum_{\nu=0}^{j} \sum_{m \in \mathbb{Z}^{n}} 2^{(\nu-j)\left(K-\alpha_{2}\right)}\left|\lambda_{v m}\right| w_{\nu}\left(2^{-v} m\right)\left(1+2^{j}\left|x-2^{-v} m\right|\right)^{\alpha-M} .
\end{aligned}
$$

Hence we obtain

$$
\begin{aligned}
& \left|\sum_{\nu=0}^{j} \sum_{m \in \mathbb{Z}^{n}} \lambda_{\nu m} w_{j}(x) \varphi_{j}(D) a_{\nu m}(x)\right| \\
& \quad \lesssim \sum_{\nu=0}^{j} 2^{-|j-\nu|\left(K-\alpha_{2}\right)}\left(\left[\eta_{\nu, \alpha^{\prime} t} *\left(\sum_{m \in \mathbb{Z}^{n}}\left|\lambda_{\nu m}\right|^{t} w_{\nu}\left(2^{-v} m\right)^{t} \chi_{\nu m}(\cdot)\right)\right](x)\right)^{1 / t}
\end{aligned}
$$

by $\alpha<M$ and Lemma 5 with $t<\min \left\{1, p^{-}, q^{-}\right\}$and $\alpha^{\prime} t>2 n$. Therefore

$$
\begin{aligned}
& \left\|\left\{f_{j}\right\}_{j=0}^{\infty}\right\|_{\ell^{q(\cdot)}\left(L^{p(\cdot)}\right)} \lesssim \|\left\{\sum_{\nu=0}^{j} 2^{-|j-v|\left(K-\alpha_{2}\right)}\right. \\
& \left.\quad \times\left(\left[\eta_{v, \alpha^{\prime} t} *\left(\sum_{m \in \mathbb{Z}^{n}}\left|\lambda_{v m}\right|^{t} w_{\nu}\left(2^{-v} m\right)^{t} \chi_{v m}\right)\right](\cdot)\right)^{\frac{1}{t}}\right\}_{j=0}^{\infty} \|_{\ell q(\cdot)\left(L^{p(\cdot)}\right)} .
\end{aligned}
$$

Using Lemma 7 and Theorem 3, we see that

$$
\begin{aligned}
\left\|\left\{f_{j}\right\}_{j=0}^{\infty}\right\|_{\ell q(\cdot)\left(L^{p(\cdot)}\right)} & \\
& \lesssim\left\|\left\{\left(\left[\eta_{v, \alpha^{\prime} t} *\left(\sum_{m \in \mathbb{Z}^{n}}\left|\lambda_{\nu m}\right|^{t} w_{\nu}\left(2^{-v} m\right)^{t} \chi_{\nu m}(\cdot)\right)\right](\cdot)\right)^{1 / t}\right\}_{\nu=0}^{\infty}\right\|_{\ell q(\cdot)\left(L^{p(\cdot)}\right)} \\
& \lesssim\left\|\left\{\sum_{m \in \mathbb{Z}^{n}}\left|\lambda_{v m}\right|^{t} w_{\nu}\left(2^{-v} m\right)^{t} \chi_{\nu m}(\cdot)\right\}_{\nu=0}^{\infty}\right\|_{\ell q(\cdot) / t\left(L^{p(\cdot) / t}\right)}^{1 / t} \\
& \lesssim\|\lambda\|_{b_{p(\cdot), q(\cdot)}^{w}} .
\end{aligned}
$$


Finally we consider

$$
\left\|\left\{f^{j}\right\}_{j=0}^{\infty}\right\|_{\ell q(\cdot)\left(L^{p(\cdot)}\right)}=\left\|\left\{\sum_{\nu=j+1}^{\infty} \sum_{m \in \mathbb{Z}^{n}} \lambda_{\nu m} w_{j}(\cdot) \varphi_{j}(D) a_{\nu m}(\cdot)\right\}_{j=0}^{\infty}\right\|_{\ell q(\cdot)\left(L^{p(\cdot)}\right)} .
$$

By Lemma 4 and the property of admissible weight sequence (Definition 2), we see that

$$
\begin{aligned}
& \left|\sum_{\nu=j+1}^{\infty} \sum_{m \in \mathbb{Z}^{n}} \lambda_{\nu m} w_{j}(x) \varphi_{j}(D) a_{v m}(x)\right| \\
& \quad \lesssim \sum_{\nu=j+1}^{\infty} \sum_{m \in \mathbb{Z}^{n}} 2^{(j-v)(L+n)}\left|\lambda_{v m}\right| w_{j}(x)\left(1+2^{j}\left|x-2^{-v} m\right|\right)^{L+n-M} \\
& \quad \lesssim \sum_{\nu=j+1}^{\infty} \sum_{m \in \mathbb{Z}^{n}} 2^{(j-v)\left(L+n+\alpha_{1}\right)}\left|\lambda_{v m}\right| w_{\nu}\left(2^{-v} m\right)\left(1+2^{j}\left|x-2^{-v} m\right|\right)^{L+n+\alpha-M},
\end{aligned}
$$

where $L+n+\alpha<M$. Using Lemma 5 with $t<\min \left\{1, p^{-}, q^{-}\right\}$and $\alpha^{\prime} t>2 n$, we obtain

$$
\begin{aligned}
& \left|\sum_{\nu=j+1}^{\infty} \sum_{m \in \mathbb{Z}^{n}} \lambda_{\nu m} w_{j}(x) \varphi_{j}(D) a_{\nu m}(x)\right| \\
& \quad \lesssim \sum_{\nu=j+1}^{\infty} 2^{-|\nu-j|\left(L+n+\alpha_{1}-n / t\right)}\left(\left[\eta_{\nu, \alpha^{\prime} t} *\left(\sum_{m \in \mathbb{Z}^{n}}\left|\lambda_{\nu m}\right|^{t} w_{\nu}\left(2^{-v} m\right)^{t} \chi_{\nu m}(\cdot)\right)\right](x)\right)^{\frac{1}{t}} .
\end{aligned}
$$

Then we have

$$
\begin{aligned}
\left\|\left\{f^{j}\right\}_{j=0}^{\infty}\right\|_{\ell q(\cdot)}\left(L^{p(\cdot)}\right) & \\
\lesssim & \|\left\{\sum_{\nu=j+1}^{\infty} 2^{-|\nu-j|\left(L+n+\alpha_{1}-n / t\right)}\right. \\
& \left.\times\left(\left[\eta_{\nu, \alpha^{\prime} t} *\left(\sum_{m \in \mathbb{Z}^{n}}\left|\lambda_{\nu m}\right|^{t} w_{\nu}\left(2^{-v} m\right)^{t} \chi_{\nu m}(\cdot)\right)\right](\cdot)\right)^{1 / t}\right\}_{j=0}^{\infty} \|_{\ell^{q(\cdot)}\left(L^{p(\cdot)}\right)} .
\end{aligned}
$$

Using Lemma 7 and Theorem 3 again, we obtain

$$
\begin{aligned}
\left\|\left\{f^{j}\right\}_{j=0}^{\infty}\right\|_{\ell q(\cdot)}\left(L^{p(\cdot)}\right) & \\
& \lesssim\left\|\left\{\left(\left[\eta_{\nu, \alpha^{\prime} t} *\left(\sum_{m \in \mathbb{Z}^{n}}\left|\lambda_{v m}\right|^{t} w_{\nu}\left(2^{-v} m\right)^{t} \chi_{v m}(\cdot)\right)\right](\cdot)\right)^{1 / t}\right\}_{\nu=0}^{\infty}\right\|_{\ell q(\cdot)\left(L^{p(\cdot)}\right)} \\
& \lesssim\left\|\left\{\sum_{m \in \mathbb{Z}^{n}}\left|\lambda_{v m}\right|^{t} w_{\nu}\left(2^{-v} m\right)^{t} \chi_{\nu m}(\cdot)\right\}_{\nu=0}^{\infty}\right\|_{\ell q(\cdot) / t\left(L^{p(\cdot) / t}\right)}^{1 / t} \\
& \lesssim\|\lambda\|_{b_{p(\cdot), q(\cdot)}^{w}} .
\end{aligned}
$$


Hence, by (17), (18) and (19), we see that $\|f\|_{B_{p(\cdot), q(\cdot)}^{w}} \lesssim\|\lambda\|_{b_{p(\cdot), q(\cdot)}^{w}}$.

\section{A multiplier theorem}

DEFINITION 6. (i) Let $\Omega$ be a compact subset of $\mathbb{R}^{n}$. Then $\mathcal{S}^{\Omega}\left(\mathbb{R}^{n}\right)$ denotes the space of $\varphi \in \mathcal{S}\left(\mathbb{R}^{n}\right)$ satisfying $\operatorname{supp} \mathcal{F} \varphi \subset \Omega$.

(ii) Let $p(\cdot) \in C^{\log }\left(\mathbb{R}^{n}\right) \cap \mathcal{P}_{0}\left(\mathbb{R}^{n}\right)$. If $\Omega=\left\{\Omega_{k}\right\}_{k=0}^{\infty}$ is a sequence of compact subsets of $\mathbb{R}^{n}$, then $L_{p(\cdot)}^{\Omega}$ is the space of all sequences $\left\{f_{k}\right\}_{k=0}^{\infty}$ of $\mathcal{S}^{\prime}\left(\mathbb{R}^{n}\right)$ such that

$$
\operatorname{supp} \mathcal{F} f_{k} \subset \Omega_{k}
$$

and $\left\|f_{k}\right\|_{L^{p(\cdot)}}<\infty$ for $k=0,1,2, \ldots$

The author generalized "the $r$ trick", to the following lemma ([13, Lemma 4.11]).

LEMma 9 [13, Lemma 4.11]. Let $r>0, v \in \mathbb{N}_{0}$ and $l \geq n+1$. Then

$$
\frac{|f(x-z)|}{\left(1+\left|2^{v} z\right|\right)^{\frac{l}{r}}} \lesssim\left(\eta_{v, l} *|f|^{r}(x)\right)^{\frac{1}{r}}
$$

holds for all $x \in \mathbb{R}^{n}, z \in \mathbb{R}^{n}$ and $f \in \mathcal{S}^{\prime}\left(\mathbb{R}^{n}\right)$ with $\operatorname{supp} \mathcal{F} f \subset\left\{\xi:|\xi| \leq 2^{v+1}\right\}$, where the implicit positive constant depends only on $r, l$ and $n$.

THEOREM 5. Let $p(\cdot), q(\cdot) \in C^{\log }\left(\mathbb{R}^{n}\right) \cap \mathcal{P}_{0}\left(\mathbb{R}^{n}\right)$ and $\boldsymbol{w}=\left\{w_{j}\right\}_{j=0}^{\infty} \in \mathcal{W}_{\alpha_{1}, \alpha_{2}}^{\alpha}\left(\mathbb{R}^{n}\right)$. Let $\Omega=\left\{\Omega_{k}\right\}_{k=0}^{\infty}$ be a sequence of compact subsets of $\mathbb{R}^{n}$ such that $\Omega_{k} \subset\left\{\xi \in \mathbb{R}^{n}\right.$ : $\left.|\xi| \leq 2^{k+1}\right\}$. If $0<r<\min \left\{p^{-}, q^{-}\right\}$and $l>2 n+2 \alpha \min \left\{p^{-}, q^{-}\right\}$, then

$$
\left\|\left\{\sup _{z \in \mathbb{R}^{n}} \frac{w_{k}(\cdot-z)\left|f_{k}(\cdot-z)\right|}{1+\left|2^{k} z\right|^{\frac{l}{r}}}\right\}_{k=0}^{\infty}\right\|\left\|_{\ell q(\cdot)\left(L^{p(\cdot)}\right)} \lesssim\right\|\left\{w_{k}(\cdot) f_{k}\right\}_{k=0}^{\infty} \|_{\ell q(\cdot)\left(L^{p(\cdot)}\right)}
$$

holds for all $\left\{f_{k}\right\}_{k=0}^{\infty} \in L_{p(\cdot)}^{\Omega}$.

PROOF. The author proved Theorem 5 in the case that $\left\{w_{k}(\cdot)\right\}_{k=0}^{\infty}=\left\{2^{k s(\cdot)}\right\}_{k=0}^{\infty}([13$, Theorem 4.13]).

Let $\left\{f_{k}\right\}_{k=0}^{\infty} \in L_{p(\cdot)}^{\Omega}$. It is easy to see that

$$
\begin{aligned}
\frac{w_{k}(x-z)\left|f_{k}(x-z)\right|}{1+\left|2^{k} z\right|^{\frac{l}{r}}} & \leq \max \left\{2^{\frac{l}{r}-1}, 1\right\} \frac{w_{k}(x-z)\left|f_{k}(x-z)\right|}{\left(1+\left|2^{k} z\right|\right)^{\frac{l}{r}}} \\
& \lesssim \max \left\{2^{\frac{l}{r}-1}, 1\right\} \frac{w_{k}(x)\left|f_{k}(x-z)\right|}{\left(1+\left|2^{k} z\right|\right)^{\frac{l-\alpha r}{r}}} \\
& \lesssim \max \left\{2^{\frac{l}{r}-1}, 1\right\} w_{k}(x)\left(\eta_{k, l-\alpha r} *\left|f_{k}\right|^{r}(x)\right)^{\frac{1}{r}} \\
& \lesssim \max \left\{2^{\frac{l}{r}-1}, 1\right\}\left(\left(\eta_{k, l-2 \alpha r}(y) * w_{k}(y)^{r}\left|f_{k}(y)\right|^{r}\right)(x)\right)^{\frac{1}{r}}
\end{aligned}
$$


for $k=0,1, \ldots$ by Lemma 9 and the property of admissible weight sequences (Definition 2). Hence we have

$$
\begin{aligned}
& \left\|\left\{\sup _{z \in \mathbb{R}^{n}} \frac{w_{k}(\cdot-z)\left|f_{k}(\cdot-z)\right|}{1+\left|2^{k} z\right|^{\frac{l}{r}}}\right\}_{k=0}^{\infty}\right\| \|_{\ell^{\frac{q(\cdot)}{r}}\left(L^{\frac{p(\cdot)}{r}}\right)} \\
& \quad \lesssim\left\|\left\{\left(\eta_{k, l-2 \alpha r}(y) * w_{k}(y)^{r}\left|f_{k}(y)\right|^{r}\right)(\cdot)\right\}_{k=0}^{\infty}\right\|_{\ell^{\frac{q(\cdot)}{r}}\left(L^{\frac{p(\cdot)}{r}}\right)}^{1 / r}
\end{aligned}
$$

Let $0<r<\min \left\{p_{-}, q_{-}\right\}$. If $l>2 n+2 \alpha r$, then we have

$$
\begin{aligned}
\left\|\left\{\sup _{z \in \mathbb{R}^{n}} \frac{w_{k}(\cdot-z)\left|f_{k}(\cdot-z)\right|}{1+\left|2^{k} z\right|^{\frac{l}{r}}}\right\}_{k=0}^{\infty}\right\|_{\ell q(\cdot)\left(L^{p(\cdot)}\right)} & \lesssim\left\|\left\{w_{k}(\cdot)^{r}\left|f_{k}\right|^{r}\right\}_{k=0}^{\infty}\right\|_{\ell^{\frac{q(\cdot)}{r}}\left(L^{\frac{p(\cdot)}{r}}\right)}^{1 / r} \\
& \sim\left\|\left\{w_{k}(\cdot) f_{k}\right\}_{k=0}^{\infty}\right\|_{\ell q(\cdot)}\left(L^{p(\cdot)}\right)
\end{aligned}
$$

by (22) and Theorem 3.

To state a multiplier theorem, we introduce the spacial Bessel-potential space $H_{2}^{v}\left(\mathbb{R}^{n}\right)$. For a real number $v$,

$$
H_{2}^{v}\left(\mathbb{R}^{n}\right)=\left\{f \in \mathcal{S}^{\prime}\left(\mathbb{R}^{n}\right):\|f\|_{H_{2}^{v}}=\left\|\left(1+|\cdot|^{2}\right)^{v / 2}(\mathcal{F} f)(\cdot)\right\|_{L^{2}}<\infty\right\} .
$$

THEOREM 6. Let $p(\cdot), q(\cdot) \in C^{\log }\left(\mathbb{R}^{n}\right) \cap \mathcal{P}_{0}\left(\mathbb{R}^{n}\right)$ and $\boldsymbol{w}=\left\{w_{j}\right\}_{j=0}^{\infty} \in \mathcal{W}_{\alpha_{1}, \alpha_{2}}^{\alpha}\left(\mathbb{R}^{n}\right)$. Let $\Omega=\left\{\Omega_{k}\right\}_{k=0}^{\infty}$ be a sequence of compact subsets of $\mathbb{R}^{n}$ such that $\Omega_{k} \subset\left\{\xi \in \mathbb{R}^{n}\right.$ : $\left.|\xi| \leq 2^{k+1}\right\}$. If $v>\frac{n}{2}+\frac{2 n+3 \alpha \min \left\{p^{-}, q^{-}\right\}}{\min \left\{p^{-}, q^{-}\right\}}$, then

$$
\begin{aligned}
\|\left\{w_{k} \mathcal{F}^{-1} M_{k} \mathcal{F} f_{k}\right\}_{k=0}^{\infty} & \|_{\ell q(\cdot)}\left(L^{p(\cdot)}\right) \\
& \lesssim \sup _{j}\left\|M_{j}\left(2^{j} \cdot\right)\right\|_{H_{2}^{v}}\left\|\left\{w_{k} f_{k}\right\}_{k=0}^{\infty}\right\|_{\ell q(\cdot)}\left(L^{p(\cdot)}\right)
\end{aligned}
$$

holds for $\left\{f_{k}\right\}_{k=0}^{\infty} \in L_{p(\cdot)}^{\Omega}$ and $\left\{M_{j}\right\}_{j=0}^{\infty} \in H_{2}^{v}\left(\mathbb{R}^{n}\right)$.

PROOF. For any $\left\{f_{k}\right\}_{k=0}^{\infty} \in L_{p(\cdot)}^{\Omega}$, the author proved

$$
\left\|f_{k}\right\|_{L^{\infty}} \lesssim\left\|f_{k}\right\|_{L^{p(\cdot)}}
$$

hods, where the implicit positive constant is independent of $\left\{f_{k}\right\}_{k=0}^{\infty}$ and $\Omega$ ([13, Theorem 4.14]). Let $\mathcal{F}^{-1} M \in L^{1}\left(\mathbb{R}^{n}\right)$. Then

$$
\left(\mathcal{F}^{-1} M \mathcal{F} f_{k}\right)(x)=c \int_{\mathbb{R}^{n}}\left(\mathcal{F}^{-1} M\right)(x-y) f_{k}(y) \mathrm{d} y
$$

makes sense for any $x \in \mathbb{R}^{n}$ by the classical Hölder inequality and (23). Here the positive constant $c$ depends only on the definition of the Fourier transform. Hence $\mathcal{F}^{-1} M_{k} \mathcal{F} f_{k}$ is well-defined. 
The author proved Theorem 6 the case that $\left\{w_{k}(\cdot)\right\}_{k=0}^{\infty}=\left\{2^{k s(\cdot)}\right\}_{k=0}^{\infty}([13$, Theorem 4.15]). We use an argument similar to the proof of [13, Theorem 4.15]. It is easy to see that

$$
\begin{aligned}
\mid w_{k}(x) & \mathcal{F}^{-1} M_{k} \mathcal{F} f_{k}(x-z) \mid \\
& \lesssim \int_{\mathbb{R}^{n}} w_{k}(x) \frac{\left|\left(\mathcal{F}^{-1} M_{k}\right)(x-z-y)\right|}{\left(1+2^{k}|x-y|\right)^{\frac{l+\alpha r}{r}}}\left|f_{k}(y)\right|\left(1+\left|2^{k}(x-y)\right|^{\frac{l+\alpha r}{r}}\right) \mathrm{d} y \\
& \lesssim \int_{\mathbb{R}^{n}} \frac{\left|\left(\mathcal{F}^{-1} M_{k}\right)(x-z-y)\right|}{\left(1+2^{k}|x-y|\right)^{\frac{l}{r}}} w_{k}(y)\left|f_{k}(y)\right|\left(1+\left|2^{k}(x-y)\right|^{\frac{l+\alpha r}{r}}\right) \mathrm{d} y
\end{aligned}
$$

by the property of admissible weight sequence (Definition 2). Hence we obtain

$$
\begin{aligned}
& \left|w_{k}(x) \mathcal{F}^{-1} M_{k} \mathcal{F} f_{k}(x-z)\right| \\
& \quad \lesssim \sup _{u \in \mathbb{R}^{n}} \frac{w_{k}(u)\left|f_{k}(u)\right|}{\left(1+2^{k}|x-u|\right)^{\frac{l}{r}}} \int_{\mathbb{R}^{n}}\left|\left(\mathcal{F}^{-1} M_{k}\right)(x-z-y)\right|\left(1+\left|2^{k}(x-y)\right|^{\frac{l+\alpha r}{r}}\right) \mathrm{d} y .
\end{aligned}
$$

Since

$$
\begin{gathered}
1+\left|2^{k}(x-y)\right|^{\frac{l+\alpha r}{r}} \lesssim\left(1+\left|2^{k}(x-y-z)\right|^{\frac{l+\alpha r}{r}}\right)\left(1+\left|2^{k} z\right|^{\frac{l+\alpha r}{r}}\right), \\
1+\left|2^{k} z\right|^{\frac{l+\alpha r}{r}} \leq 2\left(1+\left|2^{k} z\right|\right)^{\frac{l+\alpha r}{r}},
\end{gathered}
$$

the argument in the proof of $[15, \mathrm{p} 31$, Theorem] implies that

$$
\sup _{z \in \mathbb{R}^{n}} \frac{w_{k}(x)\left|\mathcal{F}^{-1} M_{k} \mathcal{F} f_{k}(x-z)\right|}{1+\left|2^{k} z\right|^{\frac{l+\alpha r}{r}}} \lesssim \sup _{z \in \mathbb{R}^{n}} \frac{w_{k}(x-z)\left|f_{k}(x-z)\right|}{1+\left|2^{k} z\right|^{\frac{l}{r}}}\left\|M_{k}\left(2^{k} \cdot\right)\right\|_{H_{2}^{v}}
$$

for $0<r<\min \left\{p^{-}, q^{-}\right\}$and $v>\frac{n}{2}+\frac{l+\alpha r}{r}$. Hence Theorem 6 is an immediate consequence of (24), by Theorem 5 and the estimate

$$
w_{k}(x)\left|\mathcal{F}^{-1} M_{k} \mathcal{F} f_{k}(x)\right| \leq \sup _{z \in \mathbb{R}^{n}} \frac{w_{k}(x)\left|\mathcal{F}^{-1} M_{k} \mathcal{F} f_{k}(x-z)\right|}{1+\left|2^{k} z\right|^{\frac{l+\alpha r}{r}}} .
$$

\section{Fatou lemma for $B_{p(\cdot), q(\cdot)}^{\boldsymbol{w}}\left(\mathbb{R}^{n}\right)$}

In this section, we prove some embedding results and completeness of the space $B_{p(\cdot), q(\cdot)}^{\boldsymbol{w}}\left(\mathbb{R}^{n}\right)$. To do this, we use the Fatou property of the norm in the variable exponent setting.

THEOREM 7 [2, Theorem 2.59]. Given $\Omega$ and $p(\cdot) \in \mathcal{P}(\Omega)$, let $\left\{f_{k}\right\}_{k=0}^{\infty} \subset L^{p(\cdot)}(\Omega)$ be a sequence of non-negative functions such that $f_{k}(x)$ is increasing and converges to a 
function $f(x)$ for almost every $x \in \Omega$. Then either $f \in L^{p(\cdot)}(\Omega)$ and $\lim _{k \rightarrow \infty}\left\|f_{k}\right\|_{L^{p(\cdot)}(\Omega)} \rightarrow$ $\|f\|_{L^{p(\cdot)}(\Omega)}$, or $f \notin L^{p(\cdot)}(\Omega)$ and $\lim _{k \rightarrow \infty}\left\|f_{k}\right\|_{L^{p(\cdot)}(\Omega)} \rightarrow \infty$.

We modify the Fatou property (Theorem 7) to apply in the $\ell^{q(\cdot)}\left(L^{p(\cdot)}\right)$ setting.

Corollary 1. Let $p(\cdot), q(\cdot) \in \mathcal{P}_{0}\left(\mathbb{R}^{n}\right)$ and $\left\{f_{k}^{q(\cdot)}\right\}_{k=0}^{\infty} \subset L^{p(\cdot) / q(\cdot)}\left(\mathbb{R}^{n}\right)$ be a sequence of non-negative functions such that $f_{k}(x)^{q(x)}$ is increasing and converges to a function $f(x)^{q(x)}$ for almost every $x \in \mathbb{R}^{n}$. Then either $f^{q(\cdot)} \in L^{p(\cdot) / q(\cdot)}\left(\mathbb{R}^{n}\right)$ and $\lim _{k \rightarrow \infty}\left\|f_{k}^{q(\cdot)}\right\|_{L^{p(\cdot) / q(\cdot)}} \rightarrow\left\|f^{q(\cdot)}\right\|_{L^{p(\cdot) / q(\cdot)}}$ or $f^{q(\cdot)} \notin L^{p(\cdot) / q(\cdot)}\left(\mathbb{R}^{n}\right)$ and $\lim _{k \rightarrow \infty}\left\|f_{k}^{q(\cdot)}\right\|_{L^{p(\cdot) / q(\cdot)}} \rightarrow$ $\infty$.

ProOF. Let $\left\{f_{k}^{q(\cdot)}\right\}_{k=0}^{\infty} \subset L^{p(\cdot) / q(\cdot)}\left(\mathbb{R}^{n}\right)$ be a sequence of non-negative functions such that $f_{k}(x)^{q(x)}$ is increasing and converges to a function $f(x)^{q(x)}$ for almost every $x \in \mathbb{R}^{n}$. If $0<r<p^{-} / q^{+}$, then $\left\{f_{k}^{r q(\cdot)}\right\}_{k=0}^{\infty} \subset L^{p(\cdot) / r q(\cdot)}\left(\mathbb{R}^{n}\right)$ is a sequence of non-negative functions such that $f_{k}(x)^{r q(x)}$ is increasing and converges to a function $f(x)^{r q(x)}$ for almost every $x \in \mathbb{R}^{n}$. By Theorem 7, we obtain that either $f^{r q(\cdot)} \in$ $L^{p(\cdot) / r q(\cdot)}\left(\mathbb{R}^{n}\right)$ and $\lim _{k \rightarrow \infty}\left\|f_{k}^{r q(\cdot)}\right\|_{L^{p(\cdot) / r q(\cdot)}} \rightarrow\left\|f^{r q(\cdot)}\right\|_{L^{p(\cdot) / r q(\cdot)}}$, or $f^{r q(\cdot)} \notin L^{p(\cdot) / r q(\cdot)}\left(\mathbb{R}^{n}\right)$ and $\lim _{k \rightarrow \infty}\left\|f_{k}^{r q(\cdot)}\right\|_{L^{p(\cdot) / r q(\cdot)}} \rightarrow \infty$. This proves this corollary.

To prove that $B_{p(\cdot), q(\cdot)}^{\boldsymbol{w}}\left(\mathbb{R}^{n}\right)$ is a quasi-Banach space, we need following Fatou lemma for $B_{p(\cdot), q(\cdot)}^{\boldsymbol{w}}\left(\mathbb{R}^{n}\right)$.

LEMMA 10. If a bounded sequence $\left\{f_{k}\right\}_{k=0}^{\infty} \subset B_{p(\cdot), q(\cdot)}^{w}\left(\mathbb{R}^{n}\right)$ converges to $f$ in the sense of $\mathcal{S}^{\prime}\left(\mathbb{R}^{n}\right)$, then $f \in B_{p(\cdot), q(\cdot)}^{w}\left(\mathbb{R}^{n}\right)$. More precisely,

$$
\|f\|_{B_{p(\cdot), q(\cdot)}^{w}} \leq \liminf _{k \rightarrow \infty}\left\|f_{k}\right\|_{B_{p(\cdot), q(\cdot)}^{w}}
$$

holds.

ProOF. Note that

$$
\mathcal{F}^{-1} \varphi_{j} * f(x)=\left\langle f, \mathcal{F}^{-1} \varphi_{j}(x-\cdot)\right\rangle=\lim _{k \rightarrow \infty}\left\langle f_{k}, \mathcal{F}^{-1} \varphi_{j}(x-\cdot)\right\rangle=\lim _{k \rightarrow \infty} \mathcal{F}^{-1} \varphi_{j} * f_{k}(x)
$$

holds for any $j \in \mathbb{N}_{0}$. Therefore, we obtain

$$
\|f\|_{B_{p(\cdot), q(\cdot)}^{w}}=\left\|\left\{w_{j}\left|\varphi_{j}(D) f\right|\right\}_{j=0}^{\infty}\right\|_{\ell^{q(\cdot)}\left(L^{p(\cdot)}\right)}=\left\|\left\{\liminf _{k \rightarrow \infty} w_{j}\left|\varphi_{j}(D) f_{k}\right|\right\}_{j=0}^{\infty}\right\|_{\ell^{q(\cdot)}\left(L^{p(\cdot)}\right)} .
$$

Firstly, we estimate the right hand side of (25). 
Since $\left\{\inf _{k \leq l} w_{j}(x)\left|\varphi_{j}(D) f_{l}(x)\right|\right\}_{k=0}^{\infty}$ is an increasing sequence for almost every $x \in$ $\mathbb{R}^{n}$, we see that

$$
\lim _{k \rightarrow \infty}\left\|\left\{\inf _{k \leq l} w_{j}\left|\varphi_{j}(D) f_{l}\right|\right\}_{j=0}^{\infty}\right\|_{\ell^{q(\cdot)}\left(L^{p(\cdot)}\right)} \leq\left\|\left\{\liminf _{k \rightarrow \infty} w_{j}\left|\varphi_{j}(D) f_{k}\right|\right\}_{j=0}^{\infty}\right\|_{\ell^{q(\cdot)}\left(L^{p(\cdot)}\right)} .
$$

Now we prove

$$
\left\|\left\{\liminf _{k \rightarrow \infty} w_{j}\left|\varphi_{j}(D) f_{k}\right|\right\}_{j=0}^{\infty}\right\|\left\|_{\ell^{q(\cdot)}\left(L^{p(\cdot)}\right)}=\lim _{k \rightarrow \infty}\right\|\left\{\inf _{k \leq l} w_{j}\left|\varphi_{j}(D) f_{l}\right|\right\}_{j=0}^{\infty} \|_{\ell^{q(\cdot)}\left(L^{p(\cdot)}\right)} .
$$

If there exists $\lambda>0$ such that

$$
\begin{aligned}
\lim _{k \rightarrow \infty}\left\|\left\{\inf _{k \leq l} w_{j}\left|\varphi_{j}(D) f_{l}\right|\right\}_{j=0}^{\infty}\right\|_{\ell^{q(\cdot)}\left(L^{p(\cdot)}\right)} & <\lambda \\
& <\left\|\left\{\liminf _{k \rightarrow \infty} w_{j}\left|\varphi_{j}(D) f_{k}\right|\right\}_{j=0}^{\infty}\right\| \|_{\ell^{q(\cdot)}\left(L^{p(\cdot)}\right)},
\end{aligned}
$$

then, by definition of $\ell^{q(\cdot)}\left(L^{p(\cdot)}\right)$ quasi norm and the Fatou property (Corollary 1), we have

$$
\begin{aligned}
1 & <\sum_{j=0}^{\infty} \|\left(\frac{\left.\lim _{\inf _{k \rightarrow \infty} w_{j}\left|\varphi_{j}(D) f_{k}\right|}\right)^{q(\cdot)} \|_{L^{p(\cdot) / q(\cdot)}}}{\lambda}=\sum_{j=0}^{\infty} \lim _{k \rightarrow \infty}\left\|\left(\frac{\inf _{k \leq l} w_{j}\left|\varphi_{j}(D) f_{l}\right|}{\lambda}\right)^{q(\cdot)}\right\|_{L^{p(\cdot) / q(\cdot)}} .\right.
\end{aligned}
$$

Since $\left\{\left\|\left(\frac{\inf _{k \leq l} w_{j}\left|\varphi_{j}(D) f_{l}\right|}{\lambda}\right)^{q(\cdot)}\right\|_{L^{p(\cdot) / q(\cdot)}}\right\}_{k=0}^{\infty}$ is an increasing sequence, we see that

$$
\begin{aligned}
\sum_{j=0}^{\infty} \lim _{k \rightarrow \infty} \| & \left\|\left(\frac{\inf _{k \leq l} w_{j}\left|\varphi_{j}(D) f_{l}\right|}{\lambda}\right)^{q(\cdot)}\right\|_{L^{p(\cdot) / q(\cdot)}} \\
& =\lim _{k \rightarrow \infty} \sum_{j=0}^{\infty}\left\|\left(\frac{\inf _{k \leq l} w_{j}\left|\varphi_{j}(D) f_{l}\right|}{\lambda}\right)^{q(\cdot)}\right\|_{L^{p(\cdot) / q(\cdot)}}
\end{aligned}
$$

holds for any $j \in \mathbb{N}_{0}$. Therefore, by (27) and (28), we obtain

$$
1<\lim _{k \rightarrow \infty} \sum_{j=0}^{\infty}\left\|\left(\frac{\inf _{k \leq l} w_{j}\left|\varphi_{j}(D) f_{l}\right|}{\lambda}\right)^{q(\cdot)}\right\|_{L^{p(\cdot) / q(\cdot)}}
$$

which implies that

$$
1<\sum_{j=0}^{\infty}\left\|\left(\frac{\inf _{k \leq l} w_{j}\left|\varphi_{j}(D) f_{l}\right|}{\lambda}\right)^{q(\cdot)}\right\|_{L^{p(\cdot) / q(\cdot)}}
$$


holds for all sufficiency large $k$, and so $\lambda<\left\|\left\{\inf _{k \leq l} w_{j}\left|\varphi_{j}(D) f_{l}\right|\right\}_{j=0}^{\infty}\right\|_{\ell^{q(\cdot)}\left(L^{p(\cdot)}\right)}$. This contradicts to (26). Therefore we have

$$
\left\|\left\{\liminf _{k \rightarrow \infty} w_{j}\left|\varphi_{j}(D) f_{k}\right|\right\}_{j=0}^{\infty}\right\|_{\ell^{q(\cdot)}\left(L^{p(\cdot)}\right)}=\lim _{k \rightarrow \infty}\left\|\left\{\inf _{k \leq l} w_{j}\left|\varphi_{j}(D) f_{l}\right|\right\}_{j=0}^{\infty}\right\|_{\ell^{q(\cdot)}\left(L^{p(\cdot)}\right)} .
$$

Finally, we estimate the right hand side of (29). For any $\lambda>0$, we see that

$$
\begin{aligned}
\sum_{j=0}^{\infty}\left\|\left(\frac{\inf _{k \leq l} w_{j}\left|\varphi_{j}(D) f_{l}\right|}{\lambda}\right)^{q(\cdot)}\right\|_{L^{p(\cdot) / q(\cdot)}} & \leq \sum_{j=0}^{\infty} \inf _{k \leq l}\left\|\left(\frac{w_{j}\left|\varphi_{j}(D) f_{l}\right|}{\lambda}\right)^{q(\cdot)}\right\|_{L^{p(\cdot) / q(\cdot)}} \\
& \leq \inf _{k \leq l} \sum_{j=0}^{\infty}\left\|\left(\frac{w_{j}\left|\varphi_{j}(D) f_{l}\right|}{\lambda}\right)^{q(\cdot)}\right\|_{L^{p(\cdot) / q(\cdot)}} .
\end{aligned}
$$

This implies that

$$
\left\|\left\{\inf _{k \leq l} w_{j}\left|\varphi_{j}(D) f_{l}\right|\right\}_{j=0}^{\infty}\right\|\left\|_{\ell^{q(\cdot)}\left(L^{p(\cdot)}\right)} \leq\right\|\left\{w_{j}\left|\varphi_{j}(D) f_{i}\right|\right\}_{j=0}^{\infty} \|_{\ell q(\cdot)\left(L^{p(\cdot)}\right)}
$$

holds for any $i \in \mathbb{N}_{0}$ with $i \geq k$. Taking the infimum over $i$ with $i \geq k$, we have

$$
\left\|\left\{\inf _{k \leq l} w_{j}\left|\varphi_{j}(D) f_{l}\right|\right\}_{j=0}^{\infty}\right\|_{\ell q(\cdot)\left(L^{p(\cdot)}\right)} \leq \inf _{k \leq l}\left\|\left\{w_{j}\left|\varphi_{j}(D) f_{l}\right|\right\}_{j=0}^{\infty}\right\|_{\ell q(\cdot)\left(L^{p(\cdot)}\right)} .
$$

By (25), (29) and (30), we see that

$$
\|f\|_{B_{p(\cdot), q(\cdot)}^{w}}^{w} \leq \liminf _{k \rightarrow \infty}\left\|\left\{w_{j}\left|\varphi_{j}(D) f_{k}\right|\right\}_{j=0}^{\infty}\right\|_{\ell q(\cdot)\left(L^{p(\cdot)}\right)}=\liminf _{k \rightarrow \infty}\left\|f_{k}\right\|_{B_{p(\cdot), q(\cdot)}^{w}} .
$$

This implies that $f \in B_{p(\cdot), q(\cdot)}^{\boldsymbol{w}}\left(\mathbb{R}^{n}\right)$.

\section{Embeddings}

The next Lemma is a certain Nikolskii inequality.

LEMMA 11. Let $p_{0}(\cdot), p_{1}(\cdot) \in C^{\log }\left(\mathbb{R}^{n}\right) \cap \mathcal{P}_{0}\left(\mathbb{R}^{n}\right)$ with $p_{1}(\cdot) \geq p_{0}(\cdot)$ and $\boldsymbol{w}=$ $\left\{w_{j}\right\}_{j=0}^{\infty} \in \mathcal{W}_{\alpha_{1}, \alpha_{2}}^{\alpha}\left(\mathbb{R}^{n}\right)$. Then the inequality

$$
\left\|\left|w_{\nu} g\right|^{q(\cdot)}\right\|_{L^{p_{1}(\cdot) / q(\cdot)}} \lesssim\left\|\left|w_{\nu} 2^{\nu\left(\frac{n}{p_{0}^{(\cdot)}}-\frac{n}{p_{1}(\cdot)}\right)} g\right|^{q(\cdot)}\right\|_{L^{p_{0}(\cdot) / q(\cdot)}}+2^{-v}
$$

holds for all $v \in \mathbb{N}_{0}, g \in \mathcal{S}^{\prime}\left(\mathbb{R}^{n}\right)$ with supp $\mathcal{F} g \subseteq\left\{\xi \in \mathbb{R}^{n}:|\xi| \leq 2^{v+1}\right\}$ and such that the norm on the right-hand side of (31) is at most one.

To prove the lemma, we need the next lemma. 
LEMma 12 [3, Lemma 6.1]. Let $s(\cdot) \in C^{\log }\left(\mathbb{R}^{n}\right)$. Then

$$
2^{k s(x)} \eta_{k, 2 l}(x-y) \lesssim 2^{k s(y)} \eta_{k, l}(x-y)
$$

holds for all $x, y \in \mathbb{R}^{n}$ and $l>C_{\log }(s)$.

Now we prove Lemma 11.

Proof of Lemma 11. Almeida and Hästö [1, Lemma 6.3] proved the lemma when $w_{\nu}(\cdot)=2^{-v s(\cdot)}$. Therefore, we use an argument similar to the proof of [1, Lemma 6.3]. Let

$$
\lambda:=\left\|\left|w_{\nu} 2^{v\left(\frac{n}{p_{0}(\cdot)}-\frac{n}{p_{1}(\cdot)}\right)} g\right|^{q(\cdot)}\right\|_{L^{p_{0}(\cdot) / q(\cdot)}}+2^{-v} .
$$

Then it suffices to prove that $\left\|\lambda^{-1 / q(\cdot)} w_{\nu} g\right\|_{L^{p_{1}(\cdot)}} \leq 1$. Using "the $r$ trick" (Lemma 3), the property of admissible weight sequence (Definition 2(i)) and Lemma 12, we see that

$$
\begin{aligned}
\lambda^{-r / q(x)} 2^{-v n r / p_{1}(x)} w_{v}^{r}|g(x)|^{r} & \lesssim \lambda^{-r / q(x)} 2^{-v n r / p_{1}(x)} w_{\nu}^{r}\left(\eta_{\nu, 2 l-r \alpha} *|g|^{r}\right)(x) \\
& \lesssim \lambda^{-r / q(x)} 2^{-v n r / p_{1}(x)}\left(\eta_{\nu, 2 l-r \alpha} *\left(w_{\nu}|g|\right)^{r}\right)(x) \\
& \lesssim \lambda^{-r / q(x)}\left(\eta_{\nu, l-r \alpha / 2} *\left(2^{-v n / p_{1}(\cdot)} w_{v}|g|\right)^{r}\right)(x)
\end{aligned}
$$

holds for large $m$. Since $\lambda \in\left[2^{-\nu}, 1+2^{-\nu}\right]$, we can move $\lambda^{-1 / q(\cdot)}$ into the convolution by Lemma 12;

$$
\begin{aligned}
\lambda^{-r / q(x)} 2^{-v n r / p_{1}(x)} w_{\nu}^{r}|g(x)|^{r} & \lesssim \lambda^{-r / q(x)}\left(\eta_{\nu, l-r \alpha / 2} *\left(2^{-v n / p_{1}(\cdot)} w_{\nu}|g|\right)^{r}\right)(x) \\
& \lesssim\left(\eta_{\nu, l / 2-r \alpha / 4} *\left(\lambda^{-1 / q(\cdot)} 2^{-v n / p_{1}(\cdot)} w_{\nu}|g|\right)^{r}\right)(x) .
\end{aligned}
$$

Let $0<r<1 /\left(p_{0}\right)^{-}$and $s(\cdot)=p_{0}(\cdot) / r \in \mathcal{P}_{0}\left(\mathbb{R}^{n}\right)$. Applying the generalized Hölder inequality to (32), we obtain

$$
\begin{aligned}
\lambda^{-r / q(x)} 2^{-v n r / p_{1}(x)} w_{v}^{r}|g(x)|^{r} \\
\quad \lesssim\left(\eta_{\nu, l / 2-r \alpha / 4} *\left(\lambda^{-1 / q(\cdot)} 2^{-v n / p_{1}(\cdot)} w_{\nu}|g|\right)^{r}\right)(x) \\
\quad \lesssim\left\|2^{-v n / s(\cdot)} \eta_{\nu, l / 2-r \alpha / 4}(x-\cdot)\right\|_{L^{s^{(\cdot)}}}^{1 / r} \| \lambda^{-1 / q(\cdot)} w_{\nu} 2^{\nu\left(\frac{n}{\left.p_{0}^{(\cdot)}-\frac{n}{p_{1}(\cdot)}\right)}|g| \|_{L^{p_{0}(\cdot)}} .\right.}
\end{aligned}
$$

By the definition of $\lambda$, the second norm on the right hand side is bounded by 1 . We check the first norm on the right hand side is also bounded. Let $t=l / 2-r \alpha / 4$. Since we can choose $l$ sufficiently large, $t$ is also sufficiently large. Then we obtain

$$
\begin{aligned}
\int_{\mathbb{R}^{n}}\left(2^{-v n / s(y)} \eta_{v, t}(x-y)\right)^{s^{\prime}(y)} \mathrm{d} y & =\int_{\mathbb{R}^{n}} 2^{v n}\left(1+2^{v}|x-y|\right)^{-t s^{\prime}(y)} \mathrm{d} y \\
& \leq \int_{\mathbb{R}^{n}}\left(1+\left|2^{v} x-z\right|\right)^{-t\left(s^{\prime}\right)^{-}} \mathrm{d} z<\infty,
\end{aligned}
$$


provided that $t\left(s^{\prime}\right)^{-}>n$. Hence we can take appropriate constant $c_{0} \in(0,1]$ such that

$$
\begin{aligned}
& \left(c_{0} \lambda^{-1 / q(x)} w_{\nu}(x)|g(x)|\right)^{p_{1}(x)} \\
& \quad=c_{0}^{p(x)}\left(c_{0} \frac{w_{\nu} 2^{-v n / p_{1}(x)}|g(x)|}{\lambda^{1 / q(x)}}\right)^{p_{1}(x)-p_{0}(x)}\left(c_{0} \frac{w_{\nu} 2^{\nu\left(\frac{n}{p_{0}(x)}-\frac{n}{p_{1}(x)}\right)}|g(x)|}{\lambda^{1 / q(x)}}\right)^{p_{0}(x)} \\
& \quad \lesssim\left(c_{0} \frac{w_{\nu} 2^{\nu\left(\frac{n}{p_{0}(x)}-\frac{n}{p_{1}(x)}\right)}|g(x)|}{\lambda^{1 / q(x)}}\right)^{p_{0}(x)} .
\end{aligned}
$$

Therefore, by integrating this inequality over $\mathbb{R}^{n}$ and by the definition of $\lambda$, we have the desired result.

In order to prove that $\mathcal{S}\left(\mathbb{R}^{n}\right)$ is dense in $B_{p(\cdot), q(\cdot)}^{\boldsymbol{w}}\left(\mathbb{R}^{n}\right)$, we use the theorem below. When $q(\cdot)$ is a constant, the theorem has been proved by Moura, Neves and Schneider [12, Theorem 2.10].

THEOREM 8. Let $\boldsymbol{w}=\left\{w_{j}\right\}_{j=0}^{\infty} \in \mathcal{W}_{\alpha_{1}, \alpha_{2}}^{\alpha}\left(\mathbb{R}^{n}\right)$ and $\boldsymbol{\rho}=\left\{\rho_{j}\right\}_{j=0}^{\infty} \in \mathcal{W}_{\beta_{1}, \beta_{2}}^{\beta}\left(\mathbb{R}^{n}\right)$.

(i) Let $p(\cdot), q_{1}(\cdot), q_{2}(\cdot) \in C^{\log }\left(\mathbb{R}^{n}\right)$ satisfy $q_{1}(\cdot) \leq q_{2}(\cdot)$. If $\frac{w_{j}(x)}{\rho_{j}(x)} \leq$ c for all $j \in \mathbb{N}_{0}$ and $x \in \mathbb{R}^{n}$, then

$$
B_{p(\cdot), q_{1}(\cdot)}^{\rho}\left(\mathbb{R}^{n}\right) \hookrightarrow B_{p(\cdot), q_{2}(\cdot)}^{\boldsymbol{w}}\left(\mathbb{R}^{n}\right) .
$$

(ii) Let $p(\cdot), q_{1}(\cdot), q_{2}(\cdot) \in C^{\log }\left(\mathbb{R}^{n}\right)$. If $\frac{w_{j}(x)}{\rho_{j}(x)} \leq c 2^{-j \varepsilon}$ for all $j \in \mathbb{N}_{0}$ and $x \in \mathbb{R}^{n}$, and some $\varepsilon>0$, then

$$
B_{p(\cdot), q_{1}(\cdot)}^{\rho}\left(\mathbb{R}^{n}\right) \hookrightarrow B_{p(\cdot), q_{2}(\cdot)}^{w}\left(\mathbb{R}^{n}\right) .
$$

(iii) Let $\boldsymbol{w}^{0}, \boldsymbol{w}^{1} \in \mathcal{W}_{\alpha_{1}, \alpha_{2}}^{\alpha}\left(\mathbb{R}^{n}\right)$ and $p_{1}(\cdot), p_{2}(\cdot) \in C^{\log }\left(\mathbb{R}^{n}\right)$ satisfy

$$
1 \leq \frac{w_{j}^{0}(x)}{w_{j}^{1}(x)}=2^{j\left(\frac{n}{p_{0}(x)}-\frac{n}{p_{1}(x)}\right)}
$$

for all $x \in \mathbb{R}^{n}$ and any $j \in \mathbb{N}_{0}$. If $q(\cdot) \in C^{\log }\left(\mathbb{R}^{n}\right)$, then

$$
B_{p_{0}(\cdot), q(\cdot)}^{\boldsymbol{w}^{0}}\left(\mathbb{R}^{n}\right) \hookrightarrow B_{p_{1}(\cdot), q(\cdot)}^{\boldsymbol{w}^{1}}\left(\mathbb{R}^{n}\right) .
$$

PROOF. To prove this theorem, we apply atomic decomposition. Therefore, we prove the embeddings for the corresponding sequence spaces. 
(i) Without loss of generality, we can assume $\|\lambda\|_{b_{p(\cdot), q_{1}(\cdot)}^{\rho}}=1$. Note that

$$
\left\|\left(\sum_{m \in \mathbb{Z}^{n}}\left|\lambda_{j m}\right| w_{j}\left(2^{-j} m\right) \chi_{j m}\right)^{q_{1}(\cdot)}\right\|_{L^{p(\cdot) / q_{1}(\cdot)}} \leq 1
$$

for any $j \in \mathbb{N}_{0}$. By Lemma 2 and Jensen's inequality, it is easy to see that

$$
\begin{aligned}
& \sum_{j=0}^{\infty}\left\|\left(\sum_{m \in \mathbb{Z}^{n}}\left|\lambda_{j m}\right| w_{j}\left(2^{-j} m\right) \chi_{j m}\right)^{q_{2}(\cdot)}\right\|_{L^{p(\cdot) / q_{2}(\cdot)}} \\
& \leq \sum_{j=0}^{\infty}\left\|\left(\sum_{m \in \mathbb{Z}^{n}}\left|\lambda_{j m}\right| \rho_{j}\left(2^{-j} m\right) \chi_{j m}\right)^{q_{1}(\cdot)}\right\|_{L^{p(\cdot) / q_{1}(\cdot)}}^{\left(q_{2} / q_{1}\right)^{-}} \\
& \leq\left(\sum_{j=0}^{\infty}\left\|\left(\sum_{m \in \mathbb{Z}^{n}}\left|\lambda_{j m}\right| \rho_{j}\left(2^{-j} m\right) \chi_{j m}\right)^{q_{1}(\cdot)}\right\|_{L^{p(\cdot) / q_{1}(\cdot)}}\right)^{\left(q_{2} / q_{1}\right)^{-}} \\
& \leq 1 .
\end{aligned}
$$

This implies that $\|\lambda\|_{b_{p(\cdot), q_{2}(\cdot)}^{w}} \lesssim\|\lambda\|_{b_{p(\cdot), q_{1}(\cdot)}^{\rho}}$, where the implicit positive constants are inde-

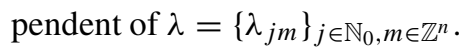

(ii) Note that $b_{p(\cdot), q_{1}(\cdot)}^{\rho}\left(\mathbb{R}^{n}\right) \hookrightarrow b_{p(\cdot), \infty}^{\rho}\left(\mathbb{R}^{n}\right)$ holds by using the normalization. Hence it is suffices to prove $b_{p(\cdot), \infty}^{\rho}\left(\mathbb{R}^{n}\right) \hookrightarrow b_{p(\cdot), q_{2}(\cdot)}^{\boldsymbol{w}}\left(\mathbb{R}^{n}\right)$. Without loss of generality, we assume $\|\lambda\|_{b_{p(\cdot), \infty}^{\rho}}^{\rho}=1$. We see that

$$
\begin{aligned}
& \sum_{j=0}^{\infty}\left\|\left(\sum_{m \in \mathbb{Z}^{n}}\left|\lambda_{j m}\right| w_{j}\left(2^{-j} m\right) \chi_{j m}\right)^{q_{2}(\cdot)}\right\|_{L^{p(\cdot) / q_{2}(\cdot)}} \\
& \leq \sum_{j=0}^{\infty} 2^{-j \varepsilon\left(q_{2}\right)^{-}}\left\|\left(\sum_{m \in \mathbb{Z}^{n}}\left|\lambda_{j m}\right| \rho_{j}\left(2^{-j} m\right) \chi_{j m}\right)^{q_{2}^{(\cdot)}}\right\|_{L^{p(\cdot) / q_{2}(\cdot)}} .
\end{aligned}
$$

By Lemma 2, we have

$$
\sum_{j=0}^{\infty}\left\|\left(\sum_{m \in \mathbb{Z}^{n}}\left|\lambda_{j m}\right| w_{j}\left(2^{-j} m\right) \chi_{j m}\right)^{q_{2}(\cdot)}\right\|_{L^{p(\cdot) / q_{2}(\cdot)}} \leq \sum_{j=0}^{\infty} 2^{-j \varepsilon\left(q_{2}\right)^{-}} .
$$

This implies that $b_{p(\cdot), \infty}^{\rho}\left(\mathbb{R}^{n}\right) \hookrightarrow b_{p(\cdot), q_{2}(\cdot)}^{\boldsymbol{w}}\left(\mathbb{R}^{n}\right)$. Hence we obtain $b_{p(\cdot), q_{1}(\cdot)}^{\boldsymbol{\rho}}\left(\mathbb{R}^{n}\right) \hookrightarrow$ $b_{p(\cdot), \infty}^{\rho}\left(\mathbb{R}^{n}\right) \hookrightarrow b_{p(\cdot), q_{2}(\cdot)}^{\boldsymbol{w}}\left(\mathbb{R}^{n}\right)$.

(iii) Without loss of generality, we can assume $\|f\|_{B_{p_{0}(\cdot), q(\cdot)}^{w^{0}}}=1$. Then, by Lemma 11, we see that

$$
\sum_{\nu=0}^{\infty}\left\|\left|w_{\nu}^{1} \varphi_{j}(D) f\right|^{q(\cdot)}\right\|_{L^{p_{1}(\cdot) / q(\cdot)}}
$$




$$
\begin{aligned}
& \lesssim \sum_{\nu=0}^{\infty}\left(\left\|\left|w_{\nu}^{1} 2^{\nu\left(\frac{n}{p_{0}(\cdot)}-\frac{n}{p_{1}(\cdot)}\right)} \varphi_{j}(D) f\right|^{q(\cdot)}\right\|_{L^{p_{0}(\cdot) / q(\cdot)}}+2^{-v}\right) \\
& \lesssim \sum_{\nu=0}^{\infty}\left(\left\|\left|w_{\nu}^{0} \varphi_{j}(D) f\right|^{q(\cdot)}\right\|_{L^{p_{0}(\cdot) / q(\cdot)}}\right)+\sum_{\nu=0}^{\infty} 2^{-v} \\
& \lesssim 1 .
\end{aligned}
$$

This implies that (iii) holds.

THEOREM 9. Let $\boldsymbol{w}=\left\{w_{j}\right\}_{j=0}^{\infty} \in \mathcal{W}_{\alpha_{1}, \alpha_{2}}^{\alpha}\left(\mathbb{R}^{n}\right), p(\cdot), q(\cdot) \in C^{\log }\left(\mathbb{R}^{n}\right) \cap \mathcal{P}_{0}\left(\mathbb{R}^{n}\right)$. Then $B_{p(\cdot), q(\cdot)}^{\boldsymbol{w}}\left(\mathbb{R}^{n}\right)$ is a quasi-Banach space and

$$
\mathcal{S}\left(\mathbb{R}^{n}\right) \hookrightarrow B_{p(\cdot), q(\cdot)}^{\boldsymbol{w}}\left(\mathbb{R}^{n}\right) \hookrightarrow \mathcal{S}^{\prime}\left(\mathbb{R}^{n}\right) .
$$

Furthermore, $\mathcal{S}\left(\mathbb{R}^{n}\right)$ is dense in $B_{p(\cdot), q(\cdot)}^{\boldsymbol{w}}\left(\mathbb{R}^{n}\right)$.

PROOF. In the case where $q(\cdot)$ equals to a constant $q<\infty$, Moura, Neves and Schneider [12, Theorem 2.11] proved this theorem.

Step 1 (Embedding). By the result of [12, Theorem 2.11], the following embeddings

$$
B_{p(\cdot), q^{+}}^{\boldsymbol{w}}\left(\mathbb{R}^{n}\right) \hookrightarrow \mathcal{S}^{\prime}\left(\mathbb{R}^{n}\right)
$$

and

$$
\mathcal{S}\left(\mathbb{R}^{n}\right) \hookrightarrow B_{p(\cdot), q^{-}}^{\boldsymbol{w}}\left(\mathbb{R}^{n}\right)
$$

hold. Therefore, by Theorem 8 (i), we get the embedding

$$
\mathcal{S}\left(\mathbb{R}^{n}\right) \hookrightarrow B_{p(\cdot), q^{-}}^{\boldsymbol{w}}\left(\mathbb{R}^{n}\right) \hookrightarrow B_{p(\cdot), q(\cdot)}^{\boldsymbol{w}}\left(\mathbb{R}^{n}\right) \hookrightarrow B_{p(\cdot), q^{+}}^{\boldsymbol{w}}\left(\mathbb{R}^{n}\right) \hookrightarrow \mathcal{S}^{\prime}\left(\mathbb{R}^{n}\right) .
$$

Step 2 (Completeness). Let $\left\{f_{k}\right\}_{k=0}^{\infty}$ be a Cauchy sequence in $B_{p(\cdot), q(\cdot)}^{\boldsymbol{w}}\left(\mathbb{R}^{n}\right)$. By $B_{p(\cdot), q(\cdot)}^{\boldsymbol{w}}\left(\mathbb{R}^{n}\right) \hookrightarrow \mathcal{S}^{\prime}\left(\mathbb{R}^{n}\right),\left\{f_{k}\right\}_{k=0}^{\infty}$ is also a Cauchy sequence in $\mathcal{S}^{\prime}\left(\mathbb{R}^{n}\right)$. Then we can find the limit element $f \in \mathcal{S}^{\prime}\left(\mathbb{R}^{n}\right)$ since $\mathcal{S}^{\prime}\left(\mathbb{R}^{n}\right)$ is complete. By Lemma 10, we have $f \in B_{p(\cdot), q(\cdot)}^{\boldsymbol{w}}\left(\mathbb{R}^{n}\right)$. Using Lemma 10 again, we see that

$$
\left\|f-f_{j}\right\|_{B_{p(\cdot), q(\cdot)}^{w}} \leq \liminf _{k \rightarrow \infty}\left\|f_{k}-f_{j}\right\|_{B_{p(\cdot), q(\cdot)}^{w}} .
$$

This means $\lim _{j \rightarrow \infty} f_{j}=f$ holds in $B_{p(\cdot), q(\cdot)}^{\boldsymbol{w}}\left(\mathbb{R}^{n}\right)$.

Step 3 (Density). We can prove the density by an argument similar to the classical case ([15, Proof of Theorem 2.3.3]).

Let $f \in B_{p(\cdot), q(\cdot)}^{w}\left(\mathbb{R}^{n}\right)$ and $\left\{\varphi_{k}\right\}_{k=0}^{\infty} \in \Phi\left(\mathbb{R}^{n}\right)$. We put

$$
f_{N}=\sum_{k=0}^{N} \varphi_{k}(D) f
$$


for $N \in \mathbb{N}_{0}$. We shall prove that $f_{N}$ converges to $f$ in the topology of $B_{p(\cdot), q(\cdot)}^{\boldsymbol{w}}\left(\mathbb{R}^{n}\right)$. Note that, for any $j \in \mathbb{N}_{0}$, there exists a positive constant $c$ such that

$$
\left\|\varphi_{j}\left(2^{j+1} \cdot\right)\right\|_{H_{2}^{v}} \leq c
$$

holds. By Theorem 6 and the estimate above, we have

$$
\begin{aligned}
\left\|f-f_{N}\right\|_{B_{p(\cdot), q(\cdot)}^{w}} & \leq c\left\|\left\{\left|\sum_{r=-1}^{1} w_{k}(\cdot)\right| \mathcal{F}^{-1} \varphi_{k} \varphi_{k+r} \mathcal{F} f \mid\right\}_{k=N}^{\infty}\right\|_{\ell q(\cdot)\left(L^{p(\cdot)}\right)} \\
& \lesssim\left\|\left\{w_{k}(\cdot) \varphi_{k}(D) f\right\}_{k=N}^{\infty}\right\|_{\ell q(\cdot)\left(L^{p(\cdot)}\right)} .
\end{aligned}
$$

Lebesgue's bounded convergence theorem implies that the right hand side of (33) tends to zero if $N \rightarrow \infty$.

Finally, for each $N \in \mathbb{N}_{0}$, we approximate $f_{N}$ by some function in $\mathcal{S}\left(\mathbb{R}^{n}\right)$. We put

$$
G_{N, \delta}=\frac{f_{N} \mathcal{F} \varphi_{0}(\delta \cdot)}{\mathcal{F} \varphi_{0}(0)}
$$

for each $N \in \mathbb{N}_{0}$. Note that $G_{N, \delta} \in \mathcal{S}\left(\mathbb{R}^{n}\right)$ because $\varphi_{0} \in \mathcal{S}\left(\mathbb{R}^{n}\right)$. Recall that $\varphi_{0}(x)=1$ for $|x| \leq 1$ and supp $\varphi_{0} \subseteq\left\{x \in \mathbb{R}^{n}:|x| \leq 2\right\}$. Hence, taking $\delta>0$ sufficiency small and applying Lebesgue's bounded theorem, we obtain

$$
\operatorname{supp} \mathcal{F}\left(f_{N}-G_{N, \delta}\right) \subset\left\{x \in \mathbb{R}^{n}:|x| \leq 2^{N+4}\right\}
$$

and

$$
\lim _{\delta \downarrow 0}\left\|f_{N}-G_{N, \delta}\right\|_{L^{p(\cdot)}}=0 .
$$

Since $\varphi_{0} \in \mathcal{S}\left(\mathbb{R}^{n}\right)$, we note that $G_{N, \delta} \in \mathcal{S}\left(\mathbb{R}^{n}\right)$, which is an approximation of $f_{N}$ in $B_{p(\cdot), q(\cdot)}^{\boldsymbol{w}}\left(\mathbb{R}^{n}\right)$. Therefore $\mathcal{S}\left(\mathbb{R}^{n}\right)$ is dense in $B_{p(\cdot), q(\cdot)}^{\boldsymbol{w}}\left(\mathbb{R}^{n}\right)$.

\section{Main theorem (Trace theorem)}

In this section, we consider a trace operator for $B_{p(\cdot), q(\cdot)}^{\boldsymbol{w}}\left(\mathbb{R}^{n}\right)$. Therefore, we assume $n \geq 2$ in this section.

We consider the trace operator

$$
\operatorname{Tr}: f\left(x^{\prime}, x_{n}\right) \longmapsto f\left(x^{\prime}, 0\right), \quad x^{\prime} \in \mathbb{R}^{n-1}, \quad f \in \mathcal{S}\left(\mathbb{R}^{n}\right) .
$$

We write $x=\left(x^{\prime}, x_{n}\right) \in \mathbb{R}^{n}$, where $x^{\prime} \in \mathbb{R}^{n-1}$ and $x_{n} \in \mathbb{R}$.

LEMMA 13 [12, Lemma 3.3]. Let $p(\cdot) \in C^{\log }\left(\mathbb{R}^{n}\right) \cap \mathcal{P}_{0}\left(\mathbb{R}^{n}\right)$ and $\boldsymbol{w} \in \mathcal{W}_{\alpha_{1}, \alpha_{2}}^{\alpha}\left(\mathbb{R}^{n}\right)$. Then $\tilde{\boldsymbol{w}}=\left\{\tilde{\boldsymbol{w}}_{j}\right\}_{j=0}^{\infty}$, defined by

$$
\tilde{w}_{j}\left(x^{\prime}\right)=w\left(x^{\prime}, 0\right) 2^{-j / \tilde{p}\left(x^{\prime}\right)}
$$


for $x^{\prime} \in \mathbb{R}^{n-1}$ and $j \in \mathbb{N}_{0}$, belongs to $\mathcal{W}_{\tilde{\alpha}_{1}, \tilde{\alpha}_{2}}^{\tilde{\alpha}}\left(\mathbb{R}^{n-1}\right)$ for $\tilde{\alpha}=\alpha+C_{\log }\left(\frac{1}{p(\cdot, 0)}\right), \tilde{\alpha}_{2}=$ $\alpha_{2}+1 / p^{-}$and $\tilde{\alpha}_{1}=\alpha_{1}-1 / p^{-}$.

As we mentioned in Section 1, Moura, Neves and Schneider [12] Proved the trace theorem for $B_{p(\cdot), q}^{w}\left(\mathbb{R}^{n}\right)$, where the summability parameter $q$ was constant. In this paper, we extend the Moura, Neves and Schneider's trace theorem for the cases where summability parameter is also variable exponent $q(\cdot)$. The next theorem is a main theorem.

THEOREM 10. Let $p(\cdot), q(\cdot) \in C^{\log }\left(\mathbb{R}^{n}\right) \cap \mathcal{P}_{0}\left(\mathbb{R}^{n}\right)$ and $\boldsymbol{w} \in \mathcal{W}_{\alpha_{1}, \alpha_{2}}^{\alpha}\left(\mathbb{R}^{n}\right)$ satisfy

$$
\alpha_{1}-\frac{1}{p^{-}}>(n-1)\left(\frac{1}{\min \left\{1, p^{-}\right\}}-1\right) .
$$

Furthermore, we write $\tilde{p}\left(x^{\prime}\right)=p\left(x^{\prime}, 0\right), \tilde{q}\left(x^{\prime}\right)=q\left(x^{\prime}, 0\right)$ and $\tilde{s}\left(x^{\prime}\right)=s\left(x^{\prime}, 0\right)$ and $\tilde{\boldsymbol{w}}=$ $\left\{\tilde{w}_{j}\right\}_{j=0}^{\infty} \in \mathcal{W}_{\tilde{\alpha_{1}}, \tilde{\alpha_{2}}}^{\tilde{\alpha}}\left(\mathbb{R}^{n-1}\right)$, where $\tilde{w}_{j}\left(x^{\prime}\right)=w\left(x^{\prime}, 0\right) 2^{-j / \tilde{p}\left(x^{\prime}\right)}, \tilde{\alpha_{1}}, \tilde{\alpha_{2}}$ and $\tilde{\alpha}$ are the same as in Lemma 13. Then the operator $\operatorname{Tr}$ can be extended to a surjective and continuous mapping from $B_{p(\cdot), q(\cdot)}^{\boldsymbol{w}}\left(\mathbb{R}^{n}\right)$ to $B_{\tilde{p}(\cdot), \tilde{q}(\cdot)}^{\tilde{w}}\left(\mathbb{R}^{n-1}\right)$.

8.1. Some lemmas for the proof of Theorem 10. To prove Theorem 10 , we need some lemmas. When we emphasize the semi-norms $\|\cdot\|_{B_{p(\cdot), q(\cdot)}^{w}}$ and $\|\cdot\|_{b_{p(\cdot), q(\cdot)}^{w}}$ on $\mathbb{R}^{n}$ or $\mathbb{R}^{n-1}$,

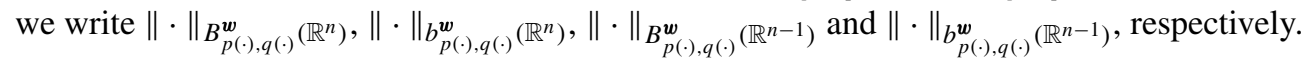

Lemma $14\left[3\right.$, Lemma 7.1]. Let $p(\cdot), q(\cdot) \in C^{\log }\left(\mathbb{R}^{n}\right) \cap \mathcal{P}_{0}\left(\mathbb{R}^{n}\right), \boldsymbol{w}=\left\{w_{j}\right\}_{j=0}^{\infty} \in$ $\mathcal{W}_{\alpha_{1}, \alpha_{2}}^{\alpha}\left(\mathbb{R}^{n}\right), \varepsilon>0$ and let $\left\{E_{v m}\right\}_{(v, m) \in \mathbb{N}_{0} \times \mathbb{Z}^{n}}$ be a collection of sets such that $E_{v m} \subset 3 Q_{v m}$ and $\left|E_{v m}\right| \geq \varepsilon\left|Q_{v m}\right|$. Further let $\chi_{E_{v m}}$ be a characteristic function on $E_{v m}$. Then we have

$$
\left\|\left\{\lambda_{v m}\right\}_{(\nu, m) \in \mathbb{N}_{0} \times \mathbb{Z}^{n}}\right\|_{b_{p(\cdot), q(\cdot)}^{w}} \sim\left\|\left\{\sum_{m \in \mathbb{Z}^{n}} w_{v}\left(2^{-v} m\right)\left|\lambda_{v m}\right| \chi_{E_{v m}}\right\}_{\nu=0}^{\infty}\right\|_{\ell q(\cdot)\left(L^{p(\cdot)}\right)}
$$

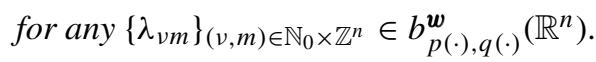

PROOF. We use an argument similar to the proof of [3, Lemma 7.1]. Note that $\chi_{v m} \lesssim$ $\eta_{v, l} * \chi_{E_{v m}}$ holds for any $l>2$. Here the implicit positive constant is independent of $v \in \mathbb{N}_{0}$ and $m \in \mathbb{Z}^{n}$. Let $0<r<\min \left\{p^{-}, q^{-}\right\}$. Then we see that

$$
\begin{aligned}
& \left\|\left\{\lambda_{v m}\right\}_{(v, m) \in \mathbb{N}_{0} \times \mathbb{Z}^{n}}\right\|_{b_{p(\cdot), q(\cdot)}^{w}} \\
& =\left\|\left\{\sum_{m \in \mathbb{Z}^{n}} w_{\nu}\left(2^{-v} m\right)\left|\lambda_{v m}\right| \chi_{v m}\right\}_{\nu=0}^{\infty}\right\|_{\ell^{q(\cdot)}\left(L^{p(\cdot)}\right)} \\
& =\left\|\left\{\sum_{m \in \mathbb{Z}^{n}} w_{\nu}\left(2^{-v} m\right)^{r}\left|\lambda_{v m}\right|^{r} \chi_{v m}\right\}_{\nu=0}^{\infty}\right\|_{\ell^{q(\cdot) / r}\left(L^{p(\cdot) / r}\right)}^{r}
\end{aligned}
$$




$$
\lesssim\left\|\left\{\eta_{\nu, l} *\left(\sum_{m \in \mathbb{Z}^{n}} w_{\nu}\left(2^{-v} m\right)^{r}\left|\lambda_{v m}\right|^{r} \chi_{E_{v m}}\right)\right\}_{\nu=0}^{\infty}\right\|_{\ell q(\cdot) / r\left(L^{p(\cdot) / r}\right)}^{r} .
$$

By Theorem 3, we obtain

$$
\begin{aligned}
\left\|\left\{\lambda_{v m}\right\}_{(v, m) \in \mathbb{N}_{0} \times \mathbb{Z}^{n}}\right\|_{b_{p(\cdot), q(\cdot)}^{\boldsymbol{w}}} & \\
& \lesssim\left\|\left\{\eta_{\nu, l} *\left(\sum_{m \in \mathbb{Z}^{n}} w_{\nu}\left(2^{-v} m\right)^{r}\left|\lambda_{v m}\right|^{r} \chi_{E_{v m}}\right)\right\}_{\nu=0}^{\infty}\right\|_{\ell q(\cdot) / r\left(L^{p(\cdot) / r}\right)}^{r} \\
& \lesssim\left\|\left\{\sum_{m \in \mathbb{Z}^{n}} w_{\nu}\left(2^{-v} m\right)^{r}\left|\lambda_{v m}\right|^{r} \chi_{E_{v m}}\right\}_{\nu=0}^{\infty}\right\|_{\ell q(\cdot) / r}\left(L^{p(\cdot) / r}\right) \\
& \lesssim\left\|\left\{\sum_{m \in \mathbb{Z}^{n}} w_{\nu}\left(2^{-v} m\right)\left|\lambda_{v m}\right| \chi_{E_{v m}}\right\}_{\nu=0}^{\infty}\right\| \|_{\ell^{q(\cdot)}\left(L^{p(\cdot)}\right)} \cdot
\end{aligned}
$$

Conversely, the opposite inequality follows from the same argument as above since $\chi_{E_{v m}} \lesssim \eta_{v, l} * \chi_{v m}$ holds, where the implicit positive constant is independent of $v \in \mathbb{N}_{0}$ and $m \in \mathbb{Z}^{n}$.

The next proposition is correspond to [12, Proposition 3.1].

Proposition 1. Let $p_{1}(\cdot), p_{2}(\cdot), q_{1}(\cdot), q_{2}(\cdot) \in C^{\log }\left(\mathbb{R}^{n}\right) \cap \mathcal{P}_{0}\left(\mathbb{R}^{n}\right)$ and $\boldsymbol{w}=$ $\left\{w_{j}\right\}_{j=0}^{\infty} \in \mathcal{W}_{\alpha_{1}, \alpha_{2}}^{\alpha}\left(\mathbb{R}^{n}\right)$. Assume that $p_{1}(\cdot)=p_{2}(\cdot)$ and $q_{1}(\cdot)=q_{2}(\cdot)$ in the upper or lower half space. For a double-index complex-valued sequence $\lambda=\left\{\lambda_{v m}\right\}_{(v, m) \in \mathbb{N}_{0} \times \mathbb{Z}^{n}}$ and for $j=0,-1$, we have

$$
\left\|\left\{\delta_{m_{n}, j} \lambda_{\nu m}\right\}_{(v, m) \in \mathbb{N}_{0} \times \mathbb{Z}^{n}}\right\|_{b_{p_{1}(\cdot), q_{1}(\cdot)}^{w}\left(\mathbb{R}^{n}\right)} \sim\left\|\left\{\delta_{m_{n}, j} \lambda_{\nu m}\right\}_{(\nu, m) \in \mathbb{N}_{0} \times \mathbb{Z}^{n}}\right\|_{b_{p_{2}(\cdot), q_{2}(\cdot)}^{w}}\left(\mathbb{R}^{n}\right) .
$$

PROOF. Firstly, we shall prove the case of $j=0$. Then we prove the case of $p_{1}(\cdot)=$ $p_{2}(\cdot)$ and $q_{1}(\cdot)=q_{2}(\cdot)$ in the lower half space because it is obvious that (36) holds if $p_{1}(\cdot)=$ $p_{2}(\cdot)$ and $q_{1}(\cdot)=q_{2}(\cdot)$ in the upper half space.

For $m=\left(m^{\prime}, 0\right) \in \mathbb{Z}^{n}$, we put

$$
E_{v m}=\left\{\left(x^{\prime}, x_{n}\right) \in \mathbb{R}^{n}:\left(x^{\prime},-x_{n}\right) \in Q_{v m},-\frac{3}{4} \cdot 2^{-v} \leq x_{n} \leq-\frac{1}{2} \cdot 2^{-v}\right\}
$$

for all other $m \in \mathbb{Z}^{n}$, we put $E_{v m}=Q_{v m}$. Since $E_{v m}$ is supported in the lower space when $m_{n}=0$, by Lemma 14 , we see that

$$
\begin{aligned}
& \left\|\left\{\delta_{m_{n}, 0} \lambda_{v m}\right\}_{(v, m) \in \mathbb{N}_{0} \times \mathbb{Z}^{n}}\right\|_{b_{p_{2}(\cdot), q_{2}(\cdot)}^{w}\left(\mathbb{R}^{n}\right)} \\
& \sim\left\|\left\{\sum_{m \in \mathbb{Z}^{n}} w_{v}\left(2^{-v} m\right) \delta_{m_{n}, 0}\left|\lambda_{v m}\right| \chi_{E_{v m}}\right\}_{\nu=0}^{\infty}\right\|_{\ell^{q_{2}(\cdot)}\left(L^{p_{2}(\cdot)}\right)}
\end{aligned}
$$




$$
\begin{aligned}
& \sim\left\|\left\{\sum_{m \in \mathbb{Z}^{n}} w_{v}\left(2^{-v} m\right) \delta_{m_{n}, 0}\left|\lambda_{v m}\right| \chi_{E_{v m}}\right\}_{\nu=0}^{\infty}\right\|_{\ell^{q_{1}(\cdot)}\left(L^{p_{1}(\cdot)}\right)} \\
& \sim\left\|\left\{\delta_{m_{n}, 0} \lambda_{v m}\right\}_{(v, m) \in \mathbb{N}_{0} \times \mathbb{Z}^{n}}\right\|_{b_{p_{1}(\cdot), q_{1}(\cdot)}^{w}\left(\mathbb{R}^{n}\right)^{\cdot}}
\end{aligned}
$$

Finally, we shall prove the case of $j=-1$. We use an argument similar to the proof [12, Proposition 3.1]. Then we prove the case of $p_{1}(\cdot)=p_{2}(\cdot)$ and $q_{1}(\cdot)=q_{2}(\cdot)$ in the upper half space because it is obvious that (36) holds if $p_{1}(\cdot)=p_{2}(\cdot)$ and $q_{1}(\cdot)=q_{2}(\cdot)$ in the lower half space. For $m=\left(m^{\prime},-1\right) \in \mathbb{Z}^{n}$, we put $E_{v m}=3 Q_{v m} \cap \mathbb{R}_{+}^{n}$; for all other $m \in \mathbb{Z}^{n}$, we put $E_{v m}=Q_{v m}$. Since $E_{v m}$ is supported in the upper space when $m_{n}=-1$, by Lemma 14, we see that

$$
\left\|\left\{\delta_{m_{n},-1} \lambda_{\nu m}\right\}_{(v, m) \in \mathbb{N}_{0} \times \mathbb{Z}^{n}}\right\|_{b_{p_{2}(\cdot), q_{2}(\cdot)}^{w}\left(\mathbb{R}^{n}\right)} \sim\left\|\left\{\delta_{m_{n},-1} \lambda_{v m}\right\}_{(\nu, m) \in \mathbb{N}_{0} \times \mathbb{Z}^{n}}\right\|_{b_{p_{1}(\cdot), q_{1}(\cdot)}^{w}\left(\mathbb{R}^{n}\right)} \cdot
$$

We extend [12, Corollary 3.2] to the next corollary.

Corollary 2 [3, Proposition 7.3]. Let $p_{1}(\cdot), p_{2}(\cdot), q_{1}(\cdot), q_{2}(\cdot) \in C^{\log }\left(\mathbb{R}^{n}\right) \cap$ $\mathcal{P}_{0}\left(\mathbb{R}^{n}\right)$ and $\boldsymbol{w}=\left\{w_{j}\right\}_{j=0}^{\infty} \in \mathcal{W}_{\alpha_{1}, \alpha_{2}}^{\alpha}\left(\mathbb{R}^{n}\right)$. Assume that $p_{1}(x)=p_{2}(x)$ and $q_{1}(x)=q_{2}(x)$ for all $x \in \mathbb{R}^{n-1} \times\{0\}$. For a double-index complex-valued sequence $\lambda=\left\{\lambda_{v m}\right\}_{(v, m) \in \mathbb{N}_{0} \times \mathbb{Z}^{n}}$ and for $j=-1,0$, we have

$$
\left\|\left\{\delta_{m_{n}, j} \lambda_{v m}\right\}_{(v, m) \in \mathbb{N}_{0} \times \mathbb{Z}^{n}}\right\|_{b_{p_{1}(\cdot), q_{1}(\cdot)}^{w}\left(\mathbb{R}^{n}\right)} \sim\left\|\left\{\delta_{m_{n}, j} \lambda_{v m}\right\}_{(\nu, m) \in \mathbb{N}_{0} \times \mathbb{Z}^{n}}\right\|_{b_{p_{2}(\cdot), q_{2}(\cdot)}^{w}}\left(\mathbb{R}^{n}\right) .
$$

Now we can remove the assumption " $p_{1}(x)=p_{2}(x)$ and $q_{1}(x)=q_{2}(x)$ for all $x \in$ $\mathbb{R}^{n-1} \times\{0\}$ ' in the Corollary 2 .

LEMMA 15. Let $p(\cdot), q(\cdot) \in C^{\log }\left(\mathbb{R}^{n}\right) \cap \mathcal{P}_{0}\left(\mathbb{R}^{n}\right)$. For a double-index complex-valued sequence $\lambda=\left\{\lambda_{v m}\right\}_{(v, m) \in \mathbb{N}_{0} \times \mathbb{Z}^{n}}$ and for $j=-1,0$, we have

$$
\left\|\left\{\lambda_{\nu\left(m^{\prime}, j\right)}\right\}_{\left(v, m^{\prime}\right) \in \mathbb{N}_{0} \times \mathbb{Z}^{n-1}}\right\|_{b_{\tilde{p}(\cdot), \tilde{q}(\cdot)}^{\tilde{w}}\left(\mathbb{R}^{n-1}\right)} \sim\left\|\left\{\delta_{m_{n}, j} \lambda_{v m}\right\}_{(v, m) \in \mathbb{N}_{0} \times \mathbb{Z}^{n}}\right\|_{b_{p(\cdot), q(\cdot)}^{w}\left(\mathbb{R}^{n}\right)} .
$$

PROOF. By Corollary 2, it suffices to consider the case that

$$
p(x)=p\left(x^{\prime}, x_{n}\right)=p\left(x^{\prime}, 0\right) \quad \text { and } \quad q(x)=q\left(x^{\prime}, x_{n}\right)-q\left(x^{\prime}, 0\right)
$$

hold for any $x=\left(x^{\prime}, x_{n}\right) \in \mathbb{R}^{n-1} \times \mathbb{R}$ with $\left|x_{n}\right| \leq 2$.

For $v \in \mathbb{N}_{0}$ and $m^{\prime} \in \mathbb{Z}^{n-1}$, let $\tilde{Q}_{v m^{\prime}}=Q_{v m^{\prime}} \times\left[2^{-v}, 2^{-v+1}\right)$ and $\chi_{\tilde{Q}_{v m}}$ be a characteristic function on $\tilde{Q}_{v m^{\prime}}$. If we can prove

$$
\begin{aligned}
& \left\|\left\{\lambda_{v\left(m^{\prime}, j\right)}\right\}_{\left(v, m^{\prime}\right) \in \mathbb{N}_{0} \times \mathbb{Z}^{n-1}}\right\|_{b_{\tilde{p}(\cdot) \tilde{q}(\cdot)}^{\tilde{w}}\left(\mathbb{R}^{n-1}\right)} \\
& =\left\|\left\{\sum_{m^{\prime} \in \mathbb{Z}^{n-1}} \tilde{w}_{\nu}\left(2^{-v} m^{\prime}\right)\left|\lambda_{\nu\left(m^{\prime}, j\right)}\right| \chi_{v m^{\prime}}\right\}_{\nu=0}^{\infty}\right\|_{\ell \tilde{q}(\cdot)\left(L^{\tilde{p}(\cdot)}\right)}
\end{aligned}
$$




$$
\sim\left\|\left\{\sum_{m^{\prime} \in \mathbb{Z}^{n-1}} w_{v}\left(2^{-v}\left(m^{\prime}, j\right)\right)\left|\lambda_{v\left(m^{\prime}, j\right)}\right| \chi_{\tilde{Q}_{v m^{\prime}}}\right\}_{\nu=0}^{\infty}\right\|_{\ell q(\cdot)\left(L^{p(\cdot)}\right)}
$$

and

$$
\begin{aligned}
& \left\|\left\{\delta_{m_{n}, j} \lambda_{v m}\right\}_{(v, m) \in \mathbb{N}_{0} \times \mathbb{Z}^{n}}\right\|_{b_{p(\cdot), q(\cdot)}^{w}\left(\mathbb{R}^{n}\right)} \\
& =\left\|\left\{\sum_{m^{\prime} \in \mathbb{Z}^{n-1}} w_{\nu}\left(2^{-v}\left(m^{\prime}, j\right)\right)\left|\lambda_{v\left(m^{\prime}, j\right)}\right| \chi_{\nu\left(m^{\prime}, j\right)}\right\}_{\nu=0}^{\infty}\right\|_{\ell q(\cdot)\left(L^{p(\cdot)}\right)} \\
& \sim\left\|\left\{\sum_{m^{\prime} \in \mathbb{Z}^{n-1}} w_{\nu}\left(2^{-v}\left(m^{\prime}, j\right)\right)\left|\lambda_{\nu\left(m^{\prime}, j\right)}\right| \chi_{\tilde{Q}_{v m^{\prime}}}\right\}_{\nu=0}^{\infty}\right\|_{\ell q(\cdot)\left(L^{p(\cdot))}\right.},
\end{aligned}
$$

then (38) and (39) imply that this lemma holds. Therefore, we prove (38) in Step 1 and (39) in Step 2.

Step 1. We prove (38). Let $\lambda>0$. Then we recall that

$$
\begin{aligned}
& \left\|\left\{\sum_{m^{\prime} \in \mathbb{Z}^{n-1}} \tilde{w}_{\nu}\left(2^{-v} m^{\prime}\right)\left|\lambda_{\nu\left(m^{\prime}, j\right)}\right| \chi_{v m^{\prime}}\right\}_{\nu=0}^{\infty}\right\|_{\ell \tilde{q}(\cdot)\left(L^{\tilde{p}(\cdot)}\right)} \\
& \quad=\inf \left\{\lambda>0: \sum_{\nu=0}^{\infty}\left\|\left(\frac{\sum_{m^{\prime} \in \mathbb{Z}^{n-1}} \tilde{w}_{\nu}\left(2^{-v} m^{\prime}\right)\left|\lambda_{\nu\left(m^{\prime}, j\right)}\right| \chi_{v m^{\prime}}}{\lambda}\right)^{\tilde{q}(\cdot)}\right\|_{L^{\tilde{p}(\cdot)}} \leq 1\right\} .
\end{aligned}
$$

Let $\mu>0$ and $\lambda_{v\left(m^{\prime}, j\right)}^{\prime}=\frac{\lambda_{\nu\left(m^{\prime}, j\right)}}{\lambda}$. Then we also recall that

$$
\left\|\left(\frac{\sum_{m^{\prime} \in \mathbb{Z}^{n-1}} \tilde{w}_{\nu}\left(2^{-v} m^{\prime}\right)\left|\lambda_{\nu\left(m^{\prime}, j\right)}\right| \chi_{\nu m^{\prime}}}{\lambda}\right)^{\tilde{q}(\cdot)}\right\|_{L} \frac{\tilde{p}(\cdot)}{\tilde{q}(\cdot)}=\inf \left\{\mu>0: I_{v} \leq 1\right\}
$$

for each $v \in \mathbb{N}_{0}$, where

$$
I_{v}=\int_{\mathbb{R}^{n-1}}\left\{\frac{\left(\sum_{m^{\prime} \in \mathbb{Z}^{n-1}} w_{v}\left(2^{-v}\left(m^{\prime}, 0\right)\right) 2^{\frac{-v}{p\left(2^{-v} m^{\prime}, 0\right)}}\left|\lambda_{v\left(m^{\prime}, j\right)}^{\prime}\right| \chi_{v m^{\prime}}\right)^{q\left(x^{\prime}, 0\right)}}{\mu}\right\}^{\frac{p\left(x^{\prime}, 0\right)}{q\left(x^{\prime}, 0\right)}} \mathrm{d} x^{\prime} .
$$

Note that, for $j=-1,0, w_{v}\left(2^{-v}\left(m^{\prime}, 0\right)\right) \sim w_{v}\left(2^{-v}\left(m^{\prime}, j\right)\right)$ holds, where the implicit positive constants depend only on $\alpha$. Using the same estimate as above, for $x^{\prime} \in Q_{v m^{\prime}}$, we have $2^{-v / p\left(2^{-v} m^{\prime}, 0\right)} \sim 2^{-v / p\left(x^{\prime}, 0\right)}$, where the implicit positive constants depend only on $C_{\log }\left(1 / p\left(\cdot^{\prime}, 0\right)\right)$. Therefore, we obtain

$$
I_{v} \sim \int_{\mathbb{R}^{n-1}} \frac{\left(\sum_{m^{\prime} \in \mathbb{Z}^{n-1}} w_{v}\left(2^{-v}\left(m^{\prime}, j\right)\right) 2^{-v / p\left(x^{\prime}, 0\right)}\left|\lambda_{v\left(m^{\prime}, j\right)}^{\prime}\right| \chi_{v m^{\prime}}\right)^{p\left(x^{\prime}, 0\right)}}{\mu^{\frac{p\left(x^{\prime}, 0\right)}{q\left(x^{\prime}, 0\right)}}} \mathrm{d} x^{\prime}
$$




$$
\sim \int_{\mathbb{R}^{n-1}} \frac{2^{-v}\left(\sum_{m^{\prime} \in \mathbb{Z}^{n-1}} w_{v}\left(2^{-v}\left(m^{\prime}, j\right)\right)\left|\lambda_{v\left(m^{\prime}, j\right)}^{\prime}\right| \chi_{v m^{\prime}}\right)^{p\left(x^{\prime}, 0\right)}}{\mu^{\frac{p\left(x^{\prime}, 0\right)}{q\left(x^{\prime}, 0\right)}}} \mathrm{d} x^{\prime} .
$$

By $2^{-v}=\int_{2^{-v}}^{2^{-v+1}} \chi_{\left[2^{-v}, 2^{-v+1}\right)}\left(x_{n}\right) \mathrm{d} x_{n}$ and $\left|x_{n}\right| \leq 2$ for any $v \in \mathbb{N}_{0}$, we see that

$$
\begin{aligned}
I_{v} & \sim \int_{\mathbb{R}^{n-1}} \frac{2^{-v}\left(\sum_{m^{\prime} \in \mathbb{Z}^{n-1}} w_{v}\left(2^{-v}\left(m^{\prime}, j\right)\right)\left|\lambda_{v\left(m^{\prime}, j\right)}^{\prime}\right| \chi_{v m^{\prime}}\right)^{p\left(x^{\prime}, 0\right)}}{\mu^{\frac{p\left(x^{\prime}, 0\right)}{q\left(x^{\prime}, 0\right)}}} \mathrm{d} x^{\prime} \\
& =\int_{\mathbb{R}^{n-1}}\left\{\int_{2^{-v}}^{2^{-v+1}} \frac{\left(\sum_{m^{\prime} \in \mathbb{Z}^{n-1}} w_{v}\left(2^{-v}\left(m^{\prime}, j\right)\right)\left|\lambda_{v\left(m^{\prime}, j\right)}^{\prime}\right| \chi_{\tilde{Q}_{v m^{\prime}}}\right)^{p\left(x^{\prime}, 0\right)}}{\mu^{\frac{p\left(x^{\prime}, 0\right)}{q\left(x^{\prime}, 0\right)}}} \mathrm{d} x_{n}\right\} \mathrm{d} x^{\prime} \\
& =\int_{\mathbb{R}^{n-1}}\left\{\int_{2^{-v}}^{2^{-v+1}} \frac{\left(\sum_{m^{\prime} \in \mathbb{Z}^{n-1}} w_{v}\left(2^{-v}\left(m^{\prime}, j\right)\right)\left|\lambda_{v\left(m^{\prime}, j\right)}^{\prime}\right| \chi_{\tilde{Q}_{v m^{\prime}}}\right)^{p\left(x^{\prime}, x_{n}\right)}}{\mu^{\frac{p\left(x^{\prime}, x_{n}\right)}{q\left(x^{\prime}, x_{n}\right)}}} \mathrm{d} x_{n}\right\} \mathrm{d} x^{\prime} .
\end{aligned}
$$

Hence we have

$$
\begin{aligned}
I_{v} & \sim \int_{\mathbb{R}^{n-1}}\left\{\int_{2^{-v}}^{2^{-v+1}} \frac{\left(\sum_{m^{\prime} \in \mathbb{Z}^{n-1}} w_{v}\left(2^{-v}\left(m^{\prime}, j\right)\right)\left|\lambda_{v\left(m^{\prime}, j\right)}^{\prime}\right| \chi_{\tilde{Q}_{v m^{\prime}}}\right)^{p\left(x^{\prime}, x_{n}\right)}}{\mu^{\frac{p\left(x^{\prime}, x_{n}\right)}{q\left(x^{\prime}, x_{n}\right)}}} \mathrm{d} x_{n}\right\} \mathrm{d} x^{\prime} \\
& =\int_{\mathbb{R}^{n}} \frac{\left(\sum_{m^{\prime} \in \mathbb{Z}^{n-1}} w_{v}\left(2^{-v}\left(m^{\prime}, j\right)\right)\left|\lambda_{v\left(m^{\prime}, j\right)}^{\prime}\right| \chi_{\tilde{Q}_{v m^{\prime}}}\right)^{p(x)}}{\mu^{\frac{p(x)}{q(x)}}} \mathrm{d} x \\
& =\int_{\mathbb{R}^{n}}\left(\frac{\left(\sum_{m^{\prime} \in \mathbb{Z}^{n-1}} w_{v}\left(2^{-v}\left(m^{\prime}, j\right)\right)\left|\lambda_{v\left(m^{\prime}, j\right)}^{\prime}\right| \chi_{\tilde{Q}_{v m^{\prime}}}\right)^{q(x)}}{\mu}\right)^{\frac{p(x)}{q(x)}} \mathrm{d} x
\end{aligned}
$$

holds for any $v \in \mathbb{N}_{0}$. This implies that

$$
\begin{aligned}
& \left\|\left(\frac{\sum_{m^{\prime} \in \mathbb{Z}^{n-1}} \tilde{w}_{v}\left(2^{-v} m^{\prime}\right)\left|\lambda_{\nu\left(m^{\prime}, j\right)}\right| \chi_{\nu m^{\prime}}}{\lambda}\right)^{\tilde{q}(\cdot)}\right\|_{L} \frac{\tilde{p}(\cdot)}{\tilde{q}(\cdot)}
\end{aligned}
$$

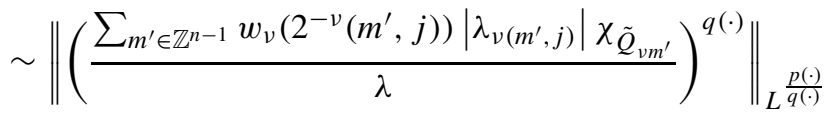

holds for any $v \in \mathbb{N}_{0}$ by (40). Therefore, we obtain (38).

Step 2. We prove (39). Firstly, we prove

$$
\left\|\left\{\sum_{m^{\prime} \in \mathbb{Z}^{n-1}} w_{\nu}\left(2^{-v}\left(m^{\prime}, j\right)\right)\left|\lambda_{v\left(m^{\prime}, j\right)}\right| \chi_{\tilde{Q}_{v m^{\prime}}}\right\}_{\nu=0}^{\infty}\right\|_{\ell q(\cdot)\left(L^{p(\cdot)}\right)}
$$




$$
\lesssim\left\|\left\{\sum_{m^{\prime} \in \mathbb{Z}^{n-1}} w_{\nu}\left(2^{-v}\left(m^{\prime}, j\right)\right)\left|\lambda_{\nu\left(m^{\prime}, j\right)}\right| \chi_{\nu\left(m^{\prime}, j\right)}\right\}_{\nu=0}^{\infty}\right\|_{\ell^{q(\cdot)}\left(L^{p(\cdot)}\right)} .
$$

Without loss of generality, we can assume $\left\|\left\{\delta_{m_{n}, j} \lambda_{v m}\right\}_{(v, m) \in \mathbb{N}_{0} \times \mathbb{Z}^{n}}\right\|_{b_{p(\cdot), q(\cdot)}^{w}\left(\mathbb{R}^{n}\right)}=1$ and $\lambda_{\nu m}=0$ when $m_{n} \neq j$. Let $\alpha:=\frac{\min \left(p^{-}, q^{-}, 1\right)}{2}$ and

$$
\lambda^{\prime}:=\left\{\lambda_{v\left(m^{\prime}, j\right)}\right\}_{\left(v, m^{\prime}\right) \in \mathbb{N}_{0} \times \mathbb{Z}^{n-1}} .
$$

If $x \in \tilde{Q}_{\nu m^{\prime}}$ and $y \in Q_{\nu\left(m^{\prime}, j\right)}$, then $|x-y| \leq 3 \sqrt{n} 2^{-v}$. Let $M>2 n$. Then we obtain

$$
1 \leq\left(\frac{3 \sqrt{n}+1}{1+2^{v}|x-y|}\right)^{M} \text {. }
$$

Hence, we see that

$$
\begin{aligned}
\left|\lambda_{v\left(m^{\prime}, j\right)}\right|^{\alpha} \chi_{\tilde{Q}_{v m^{\prime}}}(x) & \leq \frac{\chi_{\tilde{Q}_{v m^{\prime}}}(x)}{\left|Q_{v\left(m^{\prime}, j\right)}\right|} \int_{Q_{v\left(m^{\prime}, j\right)}}\left|\lambda_{v m^{\prime}}\right|^{\alpha} \chi_{\nu\left(m^{\prime}, j\right)}(y) \mathrm{d} y \\
& \lesssim \int_{\mathbb{R}^{n}} \frac{2^{v n}}{\left(1+2^{v}|x-y|\right)^{M}}\left(\left|\lambda_{\nu\left(m^{\prime}, j\right)}\right|^{\alpha} \chi_{v\left(m^{\prime}, j\right)}(y)\right) \mathrm{d} y \\
& \lesssim \int_{\mathbb{R}^{n}} \frac{2^{v n}}{\left(1+2^{v}|x-y|\right)^{M}}\left(\sum_{m^{\prime} \in \mathbb{Z}^{n-1}}\left|\lambda_{v m^{\prime}}\right|^{\alpha} \chi_{v\left(m^{\prime}, j\right)}(y)\right) \mathrm{d} y \\
& =\left(\eta_{v, M} *\left(\sum_{m^{\prime} \in \mathbb{Z}^{n-1}}\left|\lambda_{v\left(m^{\prime}, j\right)}\right| \chi_{v\left(m^{\prime}, j\right)}(\cdot)\right)^{\alpha}\right)(x) .
\end{aligned}
$$

Hence, we obtain

$$
\begin{aligned}
& \left|\sum_{m^{\prime} \in \mathbb{Z}^{n-1}} w_{\nu}\left(2^{-v}\left(m^{\prime}, j\right)\right) \lambda_{v m^{\prime}} \chi_{\tilde{Q}_{v m^{\prime}}(x)}\right| \\
& \lesssim\left(\eta_{\nu, M} *\left(\sum_{m^{\prime} \in \mathbb{Z}^{n-1}} w_{\nu}\left(2^{-v}\left(m^{\prime}, j\right)\right)\left|\lambda_{\nu\left(m^{\prime}, j\right)}\right| \chi_{\nu\left(m^{\prime}, j\right)}(\cdot)\right)^{\alpha}\right)^{\frac{1}{\alpha}}(x) .
\end{aligned}
$$

By (43) and Theorem 3, we see that

$$
\begin{aligned}
& \left\|\left\{\sum_{m^{\prime} \in \mathbb{Z}^{n-1}} w_{\nu}\left(2^{-v}\left(m^{\prime}, j\right)\right)\left|\lambda_{\nu\left(m^{\prime}, j\right)}\right| \chi_{\tilde{Q}_{v m^{\prime}}}\right\}_{\nu=0}^{\infty}\right\|_{L^{p(\cdot)(\ell q(\cdot))}} \\
& \quad \lesssim\left\|\left\{\sum_{m^{\prime} \in \mathbb{Z}^{n-1}} w_{\nu}\left(2^{-v}\left(m^{\prime}, j\right)\right)\left|\lambda_{\nu\left(m^{\prime}, j\right)}\right| \chi_{\nu\left(m^{\prime}, j\right)}\right\}_{\nu=0}^{\infty}\right\|_{L^{p(\cdot)}\left(\ell^{q(\cdot)}\right)} .
\end{aligned}
$$


Finally, we shall prove

$$
\begin{aligned}
& \left\|\left\{\sum_{m^{\prime} \in \mathbb{Z}^{n-1}} w_{\nu}\left(2^{-v}\left(m^{\prime}, j\right)\right)\left|\lambda_{\nu\left(m^{\prime}, j\right)}\right| \chi_{\nu\left(m^{\prime}, j\right)}\right\}_{\nu=0}^{\infty}\right\|_{\ell q(\cdot)\left(L^{p(\cdot)}\right)} \\
& \quad \lesssim\left\|\left\{\sum_{m^{\prime} \in \mathbb{Z}^{n-1}} w_{\nu}\left(2^{-v}\left(m^{\prime}, j\right)\right)\left|\lambda_{v\left(m^{\prime}, j\right)}\right| \chi_{\tilde{Q}_{v m^{\prime}}}\right\}_{\nu=0}^{\infty}\right\|_{\ell q(\cdot)\left(L^{p(\cdot)}\right)} .
\end{aligned}
$$

Without loss of generality, we can assume that

$$
\left\|\left\{\sum_{m^{\prime} \in \mathbb{Z}^{n-1}} w_{\nu}\left(2^{-v}\left(m^{\prime}, j\right)\right)\left|\lambda_{v\left(m^{\prime}, j\right)}\right| \chi_{\tilde{Q}_{v m^{\prime}}}\right\}_{\nu=0}^{\infty}\right\|_{\ell q(\cdot)\left(L^{p(\cdot)}\right)}=1 .
$$

By using the same argument as above, we have

$$
\begin{aligned}
\left|\lambda_{v\left(m^{\prime}, j\right)}\right|^{\alpha} \chi_{v\left(m^{\prime}, j\right)}(x) & \leq \frac{\chi_{v\left(m^{\prime}, j\right)}(x)}{\left|\tilde{Q}_{v m^{\prime}}\right|} \int_{\tilde{Q}_{v m^{\prime}}}\left|\lambda_{v m^{\prime}}\right|^{\alpha} \chi_{\tilde{Q}_{v m^{\prime}}}(y) \mathrm{d} y \\
& \lesssim \int_{\mathbb{R}^{n}} \frac{2^{v n}}{\left(1+2^{v}|x-y|\right)^{M}}\left(\left|\lambda_{\nu\left(m^{\prime}, j\right)}\right|^{\alpha} \chi_{\tilde{Q}_{v m^{\prime}}}(y)\right) \mathrm{d} y \\
& \lesssim \int_{\mathbb{R}^{n}} \frac{2^{v n}}{\left(1+2^{v}|x-y|\right)^{M}}\left(\sum_{m^{\prime} \in \mathbb{Z}^{n-1}}\left|\lambda_{v\left(m^{\prime}, j\right)}\right|^{\alpha} \chi_{\tilde{Q}_{v m^{\prime}}}(y)\right) \mathrm{d} y \\
& =\left(\eta_{v, M} *\left(\sum_{m^{\prime} \in \mathbb{Z}^{n-1}}\left|\lambda_{v\left(m^{\prime}, j\right)}\right| \chi_{\tilde{Q}_{v m^{\prime}}}(\cdot)\right)^{\alpha}\right)(x) .
\end{aligned}
$$

Hence we see that

$$
\begin{aligned}
& \left|\sum_{m^{\prime} \in \mathbb{Z}^{n-1}} w_{v}\left(2^{-v}\left(m^{\prime}, j\right)\right) \lambda_{v\left(m^{\prime}, j\right)} \chi_{v\left(m^{\prime}, j\right)}(x)\right| \\
& \quad \lesssim\left(\eta_{v, M} *\left(\sum_{m^{\prime} \in \mathbb{Z}^{n-1}} w_{v}\left(2^{-v}\left(m^{\prime}, j\right)\right)\left|\lambda_{v\left(m^{\prime}, j\right)}\right| \chi_{\tilde{Q}_{v m^{\prime}}}(\cdot)\right)^{\alpha}\right)^{\frac{1}{\alpha}}(x) .
\end{aligned}
$$

By (46) and Theorem 3, we obtain (45).

Therefore, we prove (39) by (41) and (45).

8.2. Proof of main theorem (Theorem 10). We divide the proof into 2 steps. Firstly, we prove that $\mathrm{Tr}$ is well-defined and continuous in Step 1. Next, we prove surjectivity in Step 2.

Step 1. We prove that $\mathrm{Tr}$ is well-defined and continuous. We can use a density argument. Let $f \in \mathcal{S}\left(\mathbb{R}^{n}\right)$. Since $f \in B_{p(\cdot), q(\cdot)}^{\boldsymbol{w}}\left(\mathbb{R}^{n}\right)$ by Theorem 9 , we apply the atomic decomposition 
and have

$$
f=\sum_{j=0}^{\infty} \sum_{m \in \mathbb{Z}^{n}} \lambda_{j m} a_{j m}
$$

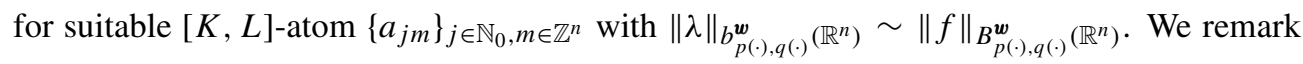
that $\operatorname{Tr}$ is well defined for $f \in \mathcal{S}\left(\mathbb{R}^{n}\right)$ from the optimal atomic decompositions (47). Furthermore, the optimal atomic decompositions that the convergence (47) holds not only in $\mathcal{S}^{\prime}\left(\mathbb{R}^{n}\right)$ but also pointwisely. Since supp $a_{j m} \subset 3 Q_{j m}, a_{j m}\left(x^{\prime}, 0\right)$ is not a zero function if and only if $m_{n}=0,-1$, where $m=\left(m_{1}, m_{2}, \ldots, m_{n}\right)$ and $x^{\prime} \in \mathbb{R}^{n-1}$. Hence, we see that

$$
\begin{aligned}
\operatorname{Tr} f\left(x^{\prime}\right) & =\sum_{j=0}^{\infty} \sum_{m \in \mathbb{Z}^{n}} \lambda_{j m} a_{j m}\left(x^{\prime}, 0\right) \\
& =\sum_{j=0}^{\infty} \sum_{m^{\prime} \in \mathbb{Z}^{n-1}} \lambda_{j\left(m^{\prime},-1\right)} a_{j\left(m^{\prime},-1\right)}\left(x^{\prime}, 0\right)+\sum_{j=0}^{\infty} \sum_{m^{\prime} \in \mathbb{Z}^{n-1}} \lambda_{j\left(m^{\prime}, 0\right)} a_{j\left(m^{\prime}, 0\right)}\left(x^{\prime}, 0\right) .
\end{aligned}
$$

Note that $\left\{a_{\nu\left(m^{\prime},-1\right)}\left({ }^{\prime}, 0\right)\right\}_{\nu \in \mathbb{N}_{0}, m^{\prime} \in \mathbb{Z}^{n-1}}$ and $\left\{a_{\nu\left(m^{\prime}, 0\right)}\left({ }^{\prime}, 0\right)\right\}_{\nu \in \mathbb{N}_{0}, m^{\prime} \in \mathbb{Z}^{n-1}}$ are $[K, L]$-atoms centered at $Q_{v m^{\prime}}$. Therefore, by Lemma 15 , we obtain

$$
\begin{aligned}
& \|\operatorname{Tr} f\|_{B_{\tilde{\tilde{p}}(\cdot), \tilde{q}(\cdot)}^{\tilde{\tilde{w}}}\left(\mathbb{R}^{n-1)}\right.}=\left\|\sum_{j=0}^{\infty} \sum_{m^{\prime} \in \mathbb{Z}^{n-1}} \lambda_{j\left(m^{\prime},-1\right)} a_{j\left(m^{\prime},-1\right)}\left(\cdot^{\prime}, 0\right)\right\|_{B_{\tilde{p}(\cdot), \tilde{q}(\cdot)}^{\tilde{w}}\left(\mathbb{R}^{n-1}\right)} \\
& +\left\|\sum_{j=0}^{\infty} \sum_{m^{\prime} \in \mathbb{Z}^{n-1}} \lambda_{j\left(m^{\prime}, 0\right)} a_{j\left(m^{\prime}, 0\right)}\left({ }^{\prime}, 0\right)\right\|_{B_{\tilde{\tilde{p}}(\cdot), \tilde{q}(\cdot)}^{\tilde{w}}\left(\mathbb{R}^{n-1}\right)}
\end{aligned}
$$

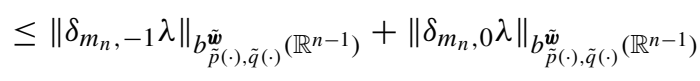

$$
\begin{aligned}
& \lesssim\left\|\delta_{m_{n},-1} \lambda\right\|_{b_{p(\cdot), q(\cdot)}^{w}\left(\mathbb{R}^{n}\right)}+\left\|\delta_{m_{n}, 0} \lambda\right\|_{b_{p(\cdot), q(\cdot)}^{w}}\left(\mathbb{R}^{n}\right) \\
& \lesssim\|f\|_{B_{p(\cdot), q(\cdot)}^{w}\left(\mathbb{R}^{n}\right)} .
\end{aligned}
$$

Let $f \in B_{p(\cdot), q(\cdot)}^{w}$. Since $\mathcal{S}\left(\mathbb{R}^{n}\right)$ is dense in $B_{p(\cdot), q(\cdot)}^{w}\left(\mathbb{R}^{n}\right)$, there exists a sequence $\left\{f_{k}\right\}_{k=0}^{\infty} \subset \mathcal{S}\left(\mathbb{R}^{n}\right)$ such that $\left\|f_{k}-f\right\|_{B_{p(\cdot), q(\cdot)}^{w}\left(\mathbb{R}^{n}\right)} \rightarrow 0$ as $k \rightarrow \infty$. Because $\left\{f_{k}\right\}_{k=0}^{\infty} \subset \mathcal{S}\left(\mathbb{R}^{n}\right)$ is a Cauchy sequence in $B_{p(\cdot), q(\cdot)}^{\boldsymbol{w}}\left(\mathbb{R}^{n}\right)$, by (48) it follows that $\left\{\operatorname{Tr} f_{k}\right\}_{k=0}^{\infty} \subset B_{\tilde{p}(\cdot), \tilde{q}(\cdot)}^{\tilde{\boldsymbol{w}}}\left(\mathbb{R}^{n-1}\right)$ is a Cauchy sequence in the complete space $B_{\tilde{p}(\cdot), \tilde{q}(\cdot)}^{\tilde{\boldsymbol{w}}}\left(\mathbb{R}^{n-1}\right)$. Therefore, there exists $g \in$ $B_{\tilde{p}(\cdot), \tilde{q}(\cdot)}^{\tilde{\tilde{w}}}\left(\mathbb{R}^{n-1}\right)$ such that $\lim _{k \rightarrow \infty} \operatorname{Tr} f_{k}=g$ in $B_{\tilde{p}(\cdot), \tilde{q}(\cdot)}^{\tilde{\tilde{w}}}\left(\mathbb{R}^{n-1}\right)$. Hence we define $\operatorname{Tr} f:=g$. We show the definition of the trace operator is well-defined. Let $\left\{h_{k}\right\}_{k=0}^{\infty} \subset \mathcal{S}\left(\mathbb{R}^{n}\right)$ is another sequence which converges to $f$ in $B_{p(\cdot), q(\cdot)}^{w}\left(\mathbb{R}^{n}\right)$. Then we see that

$$
\left\|\operatorname{Tr} h_{k}-f\right\|_{B_{\tilde{p}(\cdot), \tilde{q}(\cdot)}^{\tilde{w}}\left(\mathbb{R}^{n-1}\right)}
$$




$$
\begin{aligned}
& \lesssim\left\|\operatorname{Tr} h_{k}-\operatorname{Tr} f_{k}\right\|_{B_{\tilde{p}(\cdot), \tilde{q}(\cdot)}^{\tilde{w}}\left(\mathbb{R}^{n-1}\right)}+\left\|\operatorname{Tr} f_{k}-f\right\|_{B_{\tilde{p}(\cdot), \tilde{q}(\cdot)}^{\tilde{w}}\left(\mathbb{R}^{n-1}\right)} \\
& \lesssim\left\|h_{k}-f_{k}\right\|_{B_{p(\cdot), q(\cdot)}^{w}\left(\mathbb{R}^{n}\right)}^{w}+\left\|\operatorname{Tr} f_{k}-f\right\|_{B_{\tilde{\tilde{p}}(\cdot), \tilde{q}(\cdot)}^{\tilde{w}}\left(\mathbb{R}^{n-1}\right)} \\
& \lesssim\left\|h_{k}-f\right\|_{B_{p(\cdot), q(\cdot)}^{w}\left(\mathbb{R}^{n}\right)}^{w}+\left\|f-f_{k}\right\|_{B_{p(\cdot), q(\cdot)}^{w}}^{w}\left(\mathbb{R}^{n}\right)+\left\|\operatorname{Tr} f_{k}-f\right\|_{B_{\tilde{p}(\cdot), \tilde{q}(\cdot)}^{\tilde{w}}\left(\mathbb{R}^{n-1}\right)}
\end{aligned}
$$

Therefore, the right hand side converges to zero as $k \rightarrow \infty$. This implies that $\lim _{k \rightarrow \infty} \operatorname{Tr} h_{k}=$ $g$ in $B_{\tilde{p}(\cdot), \tilde{q}(\cdot)}^{\tilde{\boldsymbol{w}}}\left(\mathbb{R}^{n-1}\right)$. It shows that the operator $\operatorname{Tr}$ is well-defined and continuous.

Step 2. Finally, we prove surjectivity. Let $g \in \mathcal{S}\left(\mathbb{R}^{n-1}\right) \subset B_{\tilde{p}(\cdot), \tilde{q}(\cdot)}^{\tilde{\boldsymbol{w}}}\left(\mathbb{R}^{n-1}\right)$. Applying the atomic decomposition again, we have

$$
g=\sum_{j=0}^{\infty} \sum_{m^{\prime} \in \mathbb{Z}^{n-1}} \gamma_{j m^{\prime}} b_{j m^{\prime}}
$$

for suitable $[K, L]$-atom $\left\{b_{j m^{\prime}}\right\}_{j \in \mathbb{N}_{0}, m^{\prime} \in \mathbb{Z}^{n-1}}$ with

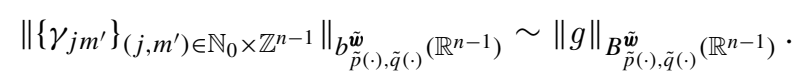

Let $\left\{b_{j m_{n}}\left(x_{n}\right)\right\}_{j \in \mathbb{N}_{0}, m_{n} \in \mathbb{Z}}$ be $[K, L]$-atoms on $\mathbb{R}$. In particular, we choose $b_{j 0}\left(x_{n}\right)$ satisfying $b_{j 0}(0)=1$. Then we define

$$
f\left(x^{\prime}, x_{n}\right)=\sum_{j=0}^{\infty} \sum_{m^{\prime} \in \mathbb{Z}^{n-1}} \delta_{m_{n}, 0} \gamma_{j m^{\prime}} b_{j m^{\prime}}\left(x^{\prime}\right) b_{j m_{n}}\left(x_{n}\right)=\sum_{j=0}^{\infty} \sum_{m \in \mathbb{Z}^{n}} \tau_{j m} a_{j m}(x),
$$

where $\tau_{j m}=\delta_{m_{n}, 0} \gamma_{j m^{\prime}}$ and $a_{j m}(x)=b_{j m^{\prime}}\left(x^{\prime}\right) b_{j m_{n}}\left(x_{n}\right)$. By the construction, we see that

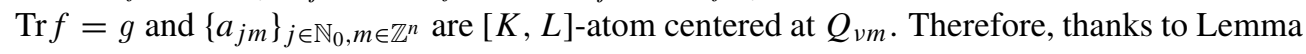
15 , we see that

$$
\begin{aligned}
\|f\|_{B_{p(\cdot), q(\cdot)}^{w}\left(\mathbb{R}^{n}\right)} & \sim\left\|\left\{\tau_{\nu m}\right\}_{(\nu, m) \in \mathbb{N}_{0} \times \mathbb{Z}^{n}}\right\|_{b_{p(\cdot), q(\cdot)}^{w}}\left(\mathbb{R}^{n}\right) \\
& \sim\left\|\left\{\tau_{\nu\left(m^{\prime}, 0\right)}\right\}_{\left(\nu, m^{\prime}\right) \in \mathbb{N}_{0} \times \mathbb{Z}^{n-1}}\right\|_{b_{\tilde{p}(\cdot), \tilde{q}(\cdot)}^{\tilde{w}}\left(\mathbb{R}^{n-1}\right)} \\
& \sim\|g\|_{B_{\tilde{p}(\cdot) \tilde{q}(\cdot)}^{\tilde{w}}\left(\mathbb{R}^{n-1}\right) \cdot} .
\end{aligned}
$$

This and the density argument imply that the operator Tr is surjective.

ACKNOWLEDGMENT. The author was partially supported by JSPS KAKENHI Grant Number 16K05212. The author would like to express his gratitude to Professor Yoshikazu Kobayashi for his reading this manuscript carefully and advices in an appropriate manner. The author would like to thank Professor Yoshihiro Sawano for his careful reading of this paper. The author is thankful to Professor Kazushi Yoshitomi and anonymous reviewer for their careful reading of this paper. 


\section{References}

[1] A. Almeida and P. HÄstö, Besov spaces with variable smoothness and integrability, J. Funct. Anal. 258 (2010), 1628-1655.

[ 2 ] D. Cruz-Uribe, SFO, A. Fiorenza, J. M. Martell and C. PÉrez, The boundedness of classical operators on variable $L^{p}$ spaces, Ann. Acad. Sci. Fenn. Math. 31 (2006), 239-264.

[ 3 ] L. Diening, P. HÄstö and S. Roudenko, Function spaces of variable smoothness and integrability, J. Funct. Anal. 256 (2009), 1731-1768.

[4] D. Drinem, Atomic decomposition of Besov spaces with variable smoothness and integrability, J. Math. Anal. Appl. 389 (2012), 15-31.

[ 5 ] M. Fraizer and B. JaWerth, Decomposition of Besov spaces, Indiana Univ. Math. J. 34 (1985), 777-799.

[6] H. KEMPKA, Local characterization of generalized 2-microlocal spaces, Jenaer Schriften zur Math. and Inf. 20/06 (2006), 38 pages.

[ 7 ] H. KempKa, 2-microlocal Besov and Triebel-Lizorkin spaces of variable integrability, Rev. Mat. Complut. 22 (2009), 227-251.

[ 8 ] H. KempKa, Atomic, molecular and wavelet decomposition of 2-microlocal Besov and Triebel-Lizorkin spaces with variable integrability, Funct. Approx. Comment. Math. 43 (2010), 171-208.

[9] H. KEMPKA and J. VyBíRAL, A note on the spaces of variable integrability and summability of Almeida and Hästö, Proc. Amer. Math. Soc. 141 (2013), 3207-3212.

[10] H. KempKa and J. Vybíral, Spaces of variable smoothness integrability: Characterizations by local means and ball means of differences, J. Fourier Anal. Appl. 18 (2012), 852-891.

[11] O. KovÁČIK and J. R'AKOSNíK, On spaces $L^{p(x)}$ and $W^{k, p(x)}$, Czech. Math. J. 41 (1991), 592-618.

[12] S. Moura, J. Neves and C. Schneider, On trace spaces of 2-microlocal Besov spaces with variable integrability, Math. Nachr. 286 (2013), 1240-1254.

[13] T. NoI, Fourier multiplier theorems for Besov and Triebel-Lizorkin spaces with variable exponents, Math. Inequal. Appl. 17 (2014), 49-74.

[14] T. NoI, Trace and extension operators for Besov spaces and Triebel-Lizorkin spaces with variable exponents, Rev. Mat. Complut. 29 (2016), 341-404.

[15] H. TRIEBEL, Theory of Function Spaces, Birkhäuser, Basel, Boston, 1983.

[16] J. XU, Variable Besov and Triebel-Lizorkin spaces, Ann. Acad. Sci. Fenn. Math. 33 (2008), 512-522.

Present Address:

DEPARTMENT OF MATHEMATICS,

CHUO UNIVERSITY,

1-13-27 KASUGA, BUNKYO-KU, TOKYO, JAPAN.

e-mail: taka.noi.hiro@gmail.com 